\title{
Optimal implementation delay of taxation with trade-off for spectrally negative Lévy risk processes
}

\author{
Wenyuan Wang ${ }^{a}$, Xueyuan $\mathrm{Wu}^{* b}$, and Cheng $\mathrm{Chi}^{c}$ \\ ${ }^{a}$ School of Mathematical Sciences, Xiamen University, Xiamen, Fujian 361005, PRC \\ ${ }^{b}$ Department of Economics, The University of Melbourne, VIC 3000, AUS \\ ${ }^{c}$ Guanghua School of Management, Peking University, Beijing 100871, PRC
}

\begin{abstract}
In this paper we consider two cases of optimal implementation delay of taxation with tradeoff under spectrally negative Lévy insurance risk processes. In the first case, we assume that the insurance company starts to pay tax only when its surplus level reaches a certain level, and at the termination time of the business there is a terminal value incurred to the company. A method is developed to determine the optimal starting-tax surplus level at which the total expected discounted value of all tax payments up to the termination time plus the discounted terminal value is maximized. In the second case, the company still pays tax subject to a starting-tax surplus level, but with capital injections to prevent bankruptcy. The total expected discounted value of tax payments minus the total discounted capital injection costs is maximized to determine the optimal starting-tax surplus level. Numerical examples are given at the end to illustrate the existence of positive optimal starting-tax surplus levels for both cases considered in this paper.
\end{abstract}

Keywords: Spectrally negative Lévy process; loss-carry-forward taxation; terminal value; capital injection; optimal implementation delay

${ }^{*}$ Corresponding author. E-mail: xueyuanw@unimelb.edu.au. 


\section{Introduction}

A well designed tax system is very important for a country's economic growth. A government can guide the flow of funds through its tax system, thereby achieving the purpose of promoting or inhibiting the development of targeting industries. The insurance industries in a country contribute a lot to the general economic growth of the society by providing stability to the functioning of process. They help to develop financial institutions and to reduce uncertainties by improving financial resources. Therefore, a sound and well-designed tax system for a country's insurance industries is not only conducive to expanding insurance demand and stimulating the development of the insurance industries, but also conducive to strengthening the government's tax income. The optimization of the insurance tax system should shift from simply focusing on cultivating and expanding tax sources to cultivating tax sources and improving the functionality of taxation. By

completing the insurance tax incentives system, the functionality of insurance taxation will be enhanced.

The functionality of taxation is mainly reflected through tax preferential policies. Tax incentives are not simple reductions or delays in tax collection. Premature tax thresholds and excessive taxation may lead to premature insolvency of insurance companies. How to achieve a perfect balance between maximizing the total amount of discounted tax payments and prolonging the lifespan of insurance companies is an important question to answer. The former objective can be achieved through optimizing the tax implementation strategies, whilst the latter one can be achieved through some custom-made financial devices, such as terminal tax payments or capital injections. Effecting a specific tax implementation strategy and a certain financial device is likely to result in an interesting trade-off that needs to be explored using actuarial modelling.

A terminal tax payment can be treated as a terminal value, which has been studied in the actuarial science literature and has been used as a tool to take account of the ruin time when studying problems like total present value of dividends until ruin. There are two potential types of terminal tax payments under the current consideration, i.e. a tax refund (a negative terminal value) or a tax payment (a positive terminal value). If the insurance company receives a terminal tax refund from the government when it is close to bankruptcy, then this tax refund has the role of a capital injection and gives the insurance company a second chance to find a way out of the financial difficulty. On the contrary, a further tax payment when the company is close to bankruptcy can be interpreted as a claw-back of early tax reliefs. One of the main observations made in this paper is that in general a terminal tax refund built in an assumed tax system helps to maximize the total discounted value of tax paid by an insurance company, whilst in an extremely low interest rate environment, a terminal tax payment can also be used as an effective financial device to maximize the total discounted tax value.

Following the objectives mentioned above, this paper aims to study the optimal implementation delay of taxation problem within the spectrally negative Lévy insurance risk framework. We assume that the taxes on an insurance company commence when the surplus level of the insurance company reaches a predetermined level $b>0$, and we employ the terminal value concept to describe a particular feature of this taxation model: a lump sum, denoted by $S$, incurred at the termination of the insurance business, which displays a time-delaying feature. This lump sum could either be an income $(S<0$, a tax benefit) or an expense $(S>0$, a claw-back of early tax relief $)$ from the insurance company's point of view. The termination is controlled by some predetermined condition, for instance the surplus level down-crossing a certain level. In this paper, we assume that the risk process is terminated whenever its net surplus level becomes negative, i.e. the risk process is terminated at the time of ruin. We will discuss the optimal choice of a starting-tax surplus 
level, denoted by $b^{*} \in[0, \infty)$, at which the taxation commences, by maximizing the total expected discounted value of all tax payments until termination plus the terminal value at termination.

The introduction of this terminal value $S$ causes some potential trade-off between maximizing the total expected discounted tax payments until ruin and maximizing the expected discounted value of $S$, when determining the optimal starting-tax surplus level $b^{*}$. In contrast to the scenario $S=0$ considered in [2], [5], [7], [9], [12] and [31], the inclusion of a negative terminal value $S<0$ will more likely lead to a trade-off between maximizing the total discounted tax payments and prolonging the life-span of the insurance company, rather than only attempting to collect taxes as much and quickly as possible. Note that we may have no choice but to sacrifice a proportion in the total discounted tax payments such that the life-span of the insurance business can be prolonged.

In this paper, we also discuss another type of trade-off within the spectrally negative Lévy insurance risk framework with tax, considering capital injections but ignoring the terminal value. [6] studied an insurance surplus process subject to taxation and capital injection through a spectrally negative Lévy process which is refracted at its running maximum and at the same time reflected from below at a certain level. [35] also considered some exit problems for spectrally negative Lévy processes reflected at either the supremum or the infimum. The capital injections can prevent the insurance company from bankruptcy at some costs, i.e. the borrowing costs on those injected capitals. We assume that the insurance company can get certain tax deductions from the capital injection costs which results in a modified value function and slightly different optimization problem, with the initial tax implementation level $a$ retaking the center of the optimization. It needs to be pointed out that the introduction of capital injection results in a trade-off between maximizing the expected accumulated discounted tax payments and minimizing the expected accumulated discounted costs of capital injection, when determining the optimal starting-tax threshold surplus level $a^{*}$. Note that decreasing tax payments can help with reducing the need of capital injections as well as lowering the costs on receiving capital injections. Hence, in contrast to the models, without capital injection, considered in [2], [5], [7], [9], [12], and [31], the maximization of the total expected discounted tax payments is no longer the sole objective when capital injection is in place.

The main contribution in this paper lies in the consideration of the above mentioned two interesting trade-off problems on optimal implementation delay of loss-carry-forward taxation under the framework of the spectrally negative Lévy processes. Taking use of some nice properties of the spectrally negative Lévy processes as well as the excursion theory of Markov processes, solutions to the objective functions of the two trade-off problems are obtained, which are concisely expressible through the scale functions associated with the underlying spectrally negative Lévy processes. Moreover, sophisticated manipulations of the objective functions are carried out to identify the optimal starting-tax surplus level, where deep understanding of the scale functions is employed. The results obtained in this paper enhance the practical relevance of the problem of taxation maximization pioneered by [5] and [7].

In recent actuarial science literature, there have been a lot of papers examining the impact of losscarry-forward taxation under an insurance risk process framework. Under the Cramér-Lundberg risk models, [2], [3], [4], [5], [12], [22], [27], [31], and [33] discussed a variety of tax-related problems. [32] considered a Markov-modulated risk model with tax, and [1] discussed the tax identity problem for Markov additive risk processes. Moreover, [2] studied a dual risk model with taxation, and [19] investigated a taxed time-homogeneous diffusion risk process. Among the above mentioned literature, [5] firstly addressed the problem of optimal implementation delay of taxation which aims to characterize the critical starting level for taxation that maximizes the expected aggregate discounted tax payments. Following the work of [5], [2] and [31] solved the taxation implementation 
delay optimization problem, respectively, for a dual risk model with exponential jumps and the Cramér-Lundberg risk model with a constant interest rate. In addition, [12] derived a necessary condition for the critical starting-tax surplus level of the optimal taxation implementation delay problem for the Cramér-Lundberg risk model with surplus-dependent premiums and tax rates.

Meanwhile, [7] solved a two-sided exit problem, the survival probability and arbitrary moments of the discounted accumulated tax payments under a general spectrally negative Lévy risk process with loss-carry-forward tax payments collected at a constant rate. The optimal starting-tax surplus level to maximize the expected discounted total tax payments was also characterized. The results of [7] were generalized in [9] by replacing the ruin stopping with a draw-down stopping. Further, [18] considered a Lévy insurance risk process with a more general tax structure, which was further generalized by [17] such that the tax rate function ranges over the whole real line. Other research papers that studied Lévy risk processes with taxation include [6], [15], [19], [24], [28], and [34]. Among the actuarial papers that considered terminal values, [25] introduced a value function which considers both expected dividends and the time value of ruin. The optimal strategy was identified for both the diffusion model and the Cramér-Lundberg model with exponential claim sizes. Meanwhile, [21] considered an optimal dividends problem with a terminal value for the spectrally negative Lévy processes with a completely monotone jump density.

This paper is organized as follows: In Section 2 we present the mathematical setup of the taxation implementation delay problem with the terminal value $S$. We derive the optimal tax implementation level $b^{*}$ given that the Lévy measure has a completely monotone density. The corresponding maximized value function is also given at $b^{*}$. In Section 3 we study the optimal implementation delay of taxation with capital injections. The optimal tax implementation level $a^{*}$ is determined as well as the corresponding maximized value function at $a^{*}$. In Section 4, we illustrate the optimal tax implementation results obtained in previous two sections using numerical examples. Concluding remarks and future research suggestions are given in Section 5. Moreover, Appendix A comprises some preliminaries concerning the spectrally negative Lévy processes. Appendix B comprises all proofs of the main results presented in previous sections.

\section{Optimal delay of taxation implementation with a terminal value}

\section{at ruin}

We assume that the before-tax surplus process for an insurance company evolves according to $X$ defined above with $X(0)=x$. In the actuarial science literature, such a surplus process is referred to as a Lévy risk process. For any given time $t \geq 0$, the insurer pays tax at a constant rate $\ell \in(0,1)$, provided that the insurer is in a profitable situation at time $t$, which is monitored by its running supremum process $\bar{X}(t)$. Hence, the cumulative taxation collected over the time interval $[0, t]$, is given by

$$
\ell(\bar{X}(t)-x), \quad t \geq 0
$$

and then the net surplus after tax at time $t$ is governed by

$$
U(t)=X(t)-\ell(\bar{X}(t)-x), \quad t \geq 0,
$$

with $U(0)=x$. A risk process with taxation similar to (1) was first considered in [5] where $X$ was a Compound Poisson process with drift, and [7] extended to a general spectrally negative Lévy 
process $X$. In those two pioneer papers [5] and [7], the arbitrary moments of the accumulated discounted tax payments and the two-sided exit problem were solved, and criteria for the surplus level for starting taxation to maximize the expected accumulated discounted tax payments were also characterized.

Define the first down-crossing time of $U$ at level 0 and the first up-crossing time of $U$ at level $b$ as

$$
\sigma_{0}^{-}:=\inf \{t \geq 0: U(t)<0\} \text { and } \sigma_{b}^{+}:=\inf \{t \geq 0: U(t)>b\}
$$

with the usual convention $\inf \emptyset:=\infty$.

The following results of the two-sided exit problem, the Laplace transform of $\sigma_{0}^{-}$and the expected accumulated discounted tax payments paid until $\sigma_{0}^{-}$can be found in [17], with a slight adaptation, for $b \in(0, \infty), x \in(0, b]$ :

$$
\begin{gathered}
\mathbb{E}_{x}\left[\mathrm{e}^{-q \sigma_{b}^{+}} \mathbf{1}_{\left\{\sigma_{b}^{+}<\sigma_{0}^{-}\right\}}\right]=\left(\frac{W_{q}(x)}{W_{q}(b)}\right)^{\frac{1}{1-\ell}}, \\
\mathbb{E}_{x}\left[\mathrm{e}^{-q \sigma_{0}^{-}} ; \sigma_{0}^{-}<\sigma_{b}^{+}\right]=\frac{1}{1-\ell} \int_{x}^{b}\left(\frac{W_{q}(x)}{W_{q}(z)}\right)^{\frac{1}{1-\ell}}\left(\frac{W_{q}^{\prime}(z)}{W_{q}(z)} Z_{q}(z)-q W_{q}(z)\right) \mathrm{d} z, \quad q \geq 0,
\end{gathered}
$$

and

$$
\mathbb{E}_{x}\left[\int_{0}^{\sigma_{b}^{+} \wedge \sigma_{0}^{-}} \mathrm{e}^{-q t} \ell \mathrm{d} \bar{X}(t)\right]=\frac{\ell}{1-\ell} \int_{x}^{b}\left(\frac{W_{q}(x)}{W_{q}(z)}\right)^{\frac{1}{1-\ell}} \mathrm{d} z, \quad q \geq 0
$$

By the arguments in Section 4.1 of [26] and (7) in Lemma 3.2 of [29], one recalls that, for $x \in(0, \infty)$, $a \in(x, \infty)$,

$$
\mathbb{E}_{x}\left[\mathrm{e}^{-q \sigma_{0}^{-}} \hbar\left(\bar{U}\left(\sigma_{0}^{-}\right)\right) ; \sigma_{0}^{-}<\sigma_{a}^{+}\right]=\frac{1}{1-\ell} \int_{x}^{a} \hbar(s)\left(\frac{W_{q}(x)}{W_{q}(s)}\right)^{\frac{1}{1-\ell}}\left(\frac{W_{q}^{\prime}(s)}{W_{q}(s)} Z_{q}(s)-q W_{q}(s)\right) \mathrm{d} s,
$$

for any measurable function $\hbar:(-\infty, \infty) \rightarrow(-\infty, \infty)$ such that the integration on the right-hand side of (5) is finite, and

$$
\begin{aligned}
\mathbb{E}_{x}\left[\mathrm{e}^{-q \sigma_{0}^{-}}\left|U\left(\sigma_{0}^{-}\right)\right| ; \sigma_{0}^{-}<\right. & \left.\sigma_{a}^{+}\right]=\frac{1}{1-\ell} \int_{x}^{a}\left(\frac{W_{q}(x)}{W_{q}(s)}\right)^{\frac{1}{1-\ell}} \\
& \times\left(Z_{q}(s)-\Psi^{\prime}(0+) W_{q}(s)-\frac{\bar{Z}_{q}(s)-\Psi^{\prime}(0+) \bar{W}_{q}(s)}{W_{q}(s)} W_{q}^{\prime}(s)\right) \mathrm{d} s .
\end{aligned}
$$

Unlike the conventional problems studied on risk models with taxation, in this section we shall take into consideration a terminal value $S \in(-\infty, \infty)$ incurred to the insurance company at the ruin time. By (3) and (4), the expected discounted tax payments made by the insurance company until ruin plus the expected discounted terminal value at the ruin time can be calculated as, for $q \geq 0, b=\infty$,

$$
\psi(x):=\mathbb{E}_{x}\left[\int_{0}^{\sigma_{0}^{-}} \mathrm{e}^{-q t} \ell \mathrm{d} \bar{X}(t)+S \mathrm{e}^{-q \sigma_{0}^{-}}\right]
$$




$$
\begin{aligned}
= & \frac{S}{1-\ell} \int_{x}^{\infty}\left(\frac{W_{q}(x)}{W_{q}(z)}\right)^{\frac{1}{1-\ell}}\left(\frac{W_{q}^{\prime}(z)}{W_{q}(z)} Z_{q}(z)-q W_{q}(z)\right) \mathrm{d} z \\
& +\frac{\ell}{1-\ell} \int_{x}^{\infty}\left(\frac{W_{q}(x)}{W_{q}(z)}\right)^{\frac{1}{1-\ell}} \mathrm{d} z, \quad x \in[0, \infty) .
\end{aligned}
$$

Inspired by [5] and [7], we presume a threshold level $b \in[0, \infty)$, which is the bar that the insurance company's surplus needs to hit before tax is payable. Then we have a modified net surplus process, for $b \in[0, \infty)$,

$$
U_{b}(t)=X(t)-\ell(\bar{X}(t) \vee b-x \vee b), \quad t \geq 0,
$$

with $U_{b}(0)=x$. The objective of this section is to determine the optimal threshold level $b^{*} \in[0, \infty)$ from which on the tax authority should collect taxes in order to maximize the expected total discounted value of tax payments plus the terminal value at ruin. For this purpose, let

$$
\sigma_{0}^{-}(b):=\inf \left\{t \geq 0 ; U_{b}(t)<0\right\},
$$

be the ruin time of $U_{b}$. In addition, define the value function $\phi(x ; b)$ with implementation delay of taxation by

$$
\phi(x ; b):=\mathbb{E}_{x}\left[\mathbf{1}_{\left\{\tau_{b}^{+}<\sigma_{0}^{-}(b)\right\}} \int_{\tau_{b}^{+}}^{\sigma_{0}^{-}(b)} \mathrm{e}^{-q t} \ell \mathrm{d} \bar{X}(t)+S \mathrm{e}^{-q \sigma_{0}^{-}(b)}\right],
$$

which, roughly speaking, is the value function of the expected discounted tax payments until ruin plus the expected discounted terminal value at ruin where taxation is delayed until the moment the risk process $U_{b}$ hits $b$. Due to the strong Markov property, (18), (3) and (4), we have, for $b \in(0, \infty)$, $x \in(0, b]$,

$$
\begin{aligned}
\phi(x ; b)= & \mathbb{E}_{x}\left[\mathbf{1}_{\left\{\tau_{b}^{+}<\tau_{0}^{-}\right\}} \int_{\tau_{b}^{+}}^{\sigma_{0}^{-}(b)} \mathrm{e}^{-q t} \ell \mathrm{d} \bar{X}(t)+S \mathrm{e}^{-q \sigma_{0}^{-}(b)} \mathbf{1}_{\left\{\tau_{b}^{+}<\sigma_{0}^{-}(b)\right\}}+S \mathrm{e}^{-q \tau_{0}^{-}} \mathbf{1}_{\left\{\tau_{0}^{-}<\tau_{b}^{+}\right\}}\right] \\
= & \mathbb{E}_{x}\left[\mathrm{e}^{-q \tau_{b}^{+}} \mathbf{1}_{\left\{\tau_{b}^{+}<\tau_{0}^{-}\right\}}\right] \mathbb{E}_{b}\left[\int_{0}^{\sigma_{0}^{-}} \mathrm{e}^{-q t} \ell \mathrm{d} \bar{X}(t)\right]+S \mathbb{E}_{x}\left[\mathrm{e}^{-q \tau_{b}^{+}} \mathbf{1}_{\left\{\tau_{b}^{+}<\sigma_{0}^{-}\right\}}\right] \mathbb{E}_{b}\left[\mathrm{e}^{-q \sigma_{0}^{-}}\right] \\
& +S \mathbb{E}_{x}\left[\mathrm{e}^{-q \tau_{0}^{-}} \mathbf{1}_{\left\{\tau_{0}^{-}<\tau_{b}^{+}\right\}}\right] \\
= & \frac{S}{1-\ell} \frac{W_{q}(x)}{W_{q}(b)} \int_{b}^{\infty}\left(\frac{W_{q}(b)}{W_{q}(z)}\right)^{\frac{1}{1-\ell}}\left(\frac{W_{q}^{\prime}(z)}{W_{q}(z)} Z_{q}(z)-q W_{q}(z)\right) \mathrm{d} z \\
& +S\left(Z_{q}(x)-\frac{W_{q}(x)}{W_{q}(b)} Z_{q}(b)\right)+\frac{\ell}{1-\ell} \frac{W_{q}(x)}{W_{q}(b)} \int_{b}^{\infty}\left(\frac{W_{q}(b)}{W_{q}(z)}\right)^{\frac{1}{1-\ell}} \mathrm{d} z \\
= & \psi(b) \frac{W_{q}(x)}{W_{q}(b)}+S\left(Z_{q}(x)-\frac{W_{q}(x)}{W_{q}(b)} Z_{q}(b)\right) .
\end{aligned}
$$

Define two auxiliary functions as follows

$$
v(b):=\psi(b)-S Z_{q}(b) \quad \text { and } \quad V(b):=\frac{W_{q}(b)}{W_{q}^{\prime}(b)} .
$$

The following result characterizes the global maximum point and the global maximum value of the value function $b \mapsto \phi(x ; b)$ for fixed $x \leq b$. It should be mentioned that a verification 
argument similar to the one in [5] is adopted. However, our arguments are more demanding since the inclusion of the terminal value brings in new challenges which require deeper understanding of the scale functions associated with the Lévy process.

Definition 2.1. The Lévy measure $\nu$ of a spectrally negative Lévy process (see, Appendix A) is said to have a completely monotone density if and only if there exists a Borel measure $\mu$ such that $\nu(\mathrm{d} x) / \mathrm{d} x=\int_{0}^{\infty} \mathrm{e}^{-x t} \mu(\mathrm{d} t)$ for all $x \in(0, \infty)$. Hence, when the Lévy measure $\nu$ has a completely monotone density, by an argument of differentiation under the integral sign one has

$$
(-1)^{n} \frac{\mathrm{d}^{n}}{\mathrm{~d} x^{n}}\left(\frac{\nu(\mathrm{d} x)}{\mathrm{d} x}\right)=\int_{0}^{\infty}(-1)^{n} \frac{\mathrm{d}^{n} \mathrm{e}^{-x t}}{\mathrm{~d} x^{n}} \mu(\mathrm{d} t)=\int_{0}^{\infty} t^{n} \mathrm{e}^{-x t} \mu(\mathrm{d} t) \geq 0, \quad n \geq 0,
$$

where the above integral is well-defined because the growth trend of the integrand $t^{n} \mathrm{e}^{-x t}$ is dominantly determined by $\mathrm{e}^{-x t}$. Actually, by Bernstein's theorem (see, Chapter XIII.4 of [14]), the Lévy measure $\nu$ has a completely monotone density if and only if $(-1)^{n} \frac{\mathrm{d}^{n}}{\mathrm{~d} x^{n}}\left(\frac{\nu(\mathrm{d} x)}{\mathrm{d} x}\right) \geq 0$ for all $n \geq 1$.

Remark 2.1. Definition 2.1 can be found in Chapter XIII.4 of [14] and is used in [5], [7], [20] and [21] as an important sufficient condition which supports their optimality results. Among particular examples fulfilling Definition 2.1 are the $\alpha$-stable processes with Lévy density

$$
\nu(\mathrm{d} x) / \mathrm{d} x=\lambda x^{-1-\alpha} \mathbf{1}_{(0, \infty)}(x), \quad \lambda>0, \alpha \in(0,2),
$$

the tempered stable process with Lévy density

$$
\nu(\mathrm{d} x) / \mathrm{d} x=\lambda x^{-1-\alpha} \mathrm{e}^{-\beta x} \mathbf{1}_{(0, \infty)}(x), \quad \lambda>0, \beta>0, \alpha \in(0,2),
$$

the Brownian motion with drift (with degenerate Lévy density 0), as well as the Cramér-Lundberg risk process with hyper exponentially distributed jumps whose Lévy density is given by

$$
\lambda \sum_{i=1}^{m} A_{i} \alpha_{i} \mathrm{e}^{-\alpha_{i} x} \mathbf{1}_{(0, \infty)}(x), \quad \lambda>0, m \in \mathbb{N}_{+}, A_{i}>0, \alpha_{i}>0, \sum_{i=1}^{m} A_{i}=1 .
$$

One can verify that all of the Lévy densities in the examples given above satisfy the condition $(-1)^{n} \frac{\mathrm{d}^{n}}{\mathrm{~d} x^{n}}\left(\frac{\nu(\mathrm{d} x)}{\mathrm{d} x}\right) \geq 0$, for all $n \geq 1$, so that they are all completely monotone.

Theorem 2.1. Assume that the Lévy measure has a completely monotone density.

If $v(0)>V(0)\left(1-S q W_{q}(0)\right)$, then there is a unique positive solution $b^{+}$of the following equation

$$
v(b)-V(b)\left(1-S q W_{q}(b)\right)=0 \Leftrightarrow v^{\prime}(b)=1-S q W_{q}(b),
$$


and the function $\phi(x ; b)$ attains its global maximum at $b^{*}=b^{+}$. Otherwise, if $v(0) \leq V(0)(1-$ $\left.S q W_{q}(0)\right)$, then $(10)$ has no positive solution, and the function $\phi(x ; b)$ attains its global maximum at $b^{*}=0$.

Moreover, we have

$$
\phi\left(x ; b^{*}\right)=S Z_{q}(x)+\frac{W_{q}(x)}{W_{q}^{\prime}\left(b^{*}\right)}\left(1-S q W_{q}\left(b^{*}\right)\right) .
$$

Proof. The proof of this theorem is given in Appendix B.

Remark 2.2. The assumption that the Lévy measure of $X$ has a completely monotone density was firstly introduced in [21] to identify a sufficient condition, under which a barrier dividend strategy is the optimal dividend strategy producing the largest expected total discounted dividend payments. Also, with this assumption, the scale functions have some desirable analytic properties.

Remark 2.3. Note that if we have $b^{*}<x$, then the optimal starting-tax surplus level should be $x$ itself.

Remark 2.4. When $S=0$, we can see that

$$
\phi(x ; b)=\frac{W_{q}(x)}{W_{q}(b)} \psi(b), \quad v(b)=\psi(b)=\frac{\ell}{1-\ell} \int_{b}^{\infty}\left(\frac{W_{q}(b)}{W_{q}(z)}\right)^{\frac{1}{1-\ell}} \mathrm{d} z,
$$

and $\frac{\partial \phi(x ; b)}{\partial b}=0$ is equivalent to

$$
v(b)=V(b) \quad \text { or } \quad v^{\prime}(b)=1
$$

which has at most one positive solution. If $v(0) \leq V(0)$, then the map $b \mapsto \phi(x ; b)$ attains its global maximum at $b^{*}=0$. If $v(0)>V(0)$, the map $b \mapsto \phi(x ; b)$ attains its global maximum at the positive solution $b^{*}$ of $(10)$. Thus, the global maximum value of $\phi(x ; b)$ is given by

$$
\phi\left(x ; b^{*}\right)=\frac{W_{q}(x)}{W_{q}\left(b^{*}\right)} v\left(b^{*}\right)=\frac{W_{q}(x)}{W_{q}^{\prime}\left(b^{*}\right)} .
$$

These results coincide with the ones obtained in [5].

A numerical example is given in Section 4 to illustrate the existence of positive optimal tax implementation threshold as given in Theorem 2.1 with discussions from the practical point of view. 


\section{Optimal delay of taxation implementation with penalized capi-} tal injection

This section is devoted to studying the optimal implementation delay of taxation for a Lévy risk process with capital injections that reflect the risk process at its infimum (or, at 0). Taking capital injections into consideration under the Lévy risk process, one can see that the surplus process never goes bankrupt, and hence taxation payments are expected to be collectible up to $\infty$ rather than up to the time of ruin as in the case without capital injections. No terminal value will be considered in this section.

In [6], a risk process refracted from both above and below with rates less than 1 was defined path by path through a recursive algorithm. The risk process $\{R(t) ; t \geq 0\}$ with taxation and capital injections, which serves as the main object of this section as well as in [6], is actually a risk process that is refracted from above with rate $\ell<1$ and refracted from below with rate 1 . In order to better develop our arguments, we re-define the risk process $\{R(t) ; t \geq 0\}$ in a more transparent way as follows.

- Let $\sigma_{0,1}^{-}=\sigma_{0}^{-}$and $U_{1}(t)=U(t)$ for $t \geq 0$. If $\sigma_{0,1}^{-}<\infty$, then define

$$
R_{1}(t)=X(t)-X\left(\sigma_{0,1}^{-}\right)-\inf _{s \in\left[\sigma_{0,1}^{-}, t\right]}\left(X(t)-X\left(\sigma_{0,1}^{-}\right)\right) \wedge 0, \quad t \in\left[\sigma_{0,1}^{-}, \infty\right),
$$

and

$$
\mathrm{H}_{1}=\sup _{0 \leq s<\sigma_{0,1}^{-}} U_{1}(s), \quad \sigma_{\mathrm{H}_{1}, 1}^{+}=\inf \left\{t \geq \sigma_{0,1}^{-} ; R_{1}(t)>\mathrm{H}_{1}\right\}
$$

Define the risk process $R$ over the time interval $\left[0, \sigma_{\mathrm{H}_{1}, 1}^{+}\right)$as

$$
\begin{aligned}
& R(t)=U_{1}(t), \quad \text { if } \quad 0 \leq t<\sigma_{0,1}^{-}, \\
& R(t)=R_{1}(t), \quad \text { if } \quad \sigma_{0,1}^{-} \leq t<\sigma_{\mathrm{H}_{1}, 1}^{+} \text {and } \sigma_{0,1}^{-}<\infty .
\end{aligned}
$$

- For $n \geq 1$, if $\sigma_{\mathrm{H}_{n}, n}^{+}<\infty$ (or, equivalently, $\sigma_{0, n}^{-}<\infty$ ), let

$$
\begin{aligned}
U_{n+1}(t)= & \mathrm{H}_{n}+X(t)-X\left(\sigma_{\mathrm{H}_{n}, n}^{+}\right) \\
& -\ell\left(\sup _{\sigma_{\mathrm{H}_{n}, n}^{+} \leq s \leq t} X(s)-X\left(\sigma_{\mathrm{H}_{n}, n}^{+}\right)\right), \quad t \in\left[\sigma_{\mathrm{H}_{n}, n}^{+}, \infty\right),
\end{aligned}
$$

and

$$
\sigma_{0, n+1}^{-}=\inf \left\{t \geq \sigma_{\mathrm{H}_{n}, n}^{+} ; U_{n+1}(t)<0\right\}, \quad \mathrm{H}_{n+1}=\sup _{\sigma_{\mathrm{H}_{n}, n}^{+} \leq s<\sigma_{0, n+1}^{-}} U_{n+1}(s),
$$

with the convention of $\inf \emptyset=\infty$. If $\sigma_{0, n+1}^{-}<\infty$, let further

$$
R_{n+1}(t)=X(t)-X\left(\sigma_{0, n+1}^{-}\right)-\inf _{s \in\left[\sigma_{0, n+1}^{-}, t\right]}\left(X(t)-X\left(\sigma_{0, n+1}^{-}\right)\right) \wedge 0, \quad t \in\left[\sigma_{0, n+1}^{-}, \infty\right),
$$

and

$$
\sigma_{\mathrm{H}_{n+1}, n+1}^{+}=\inf \left\{t \geq \sigma_{0, n+1}^{-} ; R_{n+1}(t)>\mathrm{H}_{n+1}\right\} .
$$


Accordingly, define the risk process $R$ over the time interval $\left[\sigma_{\mathrm{H}_{n}, n}^{+}, \sigma_{\mathrm{H}_{n+1}, n+1}^{+}\right)$as

$$
\begin{aligned}
& R(t)=U_{n+1}(t), \quad \text { if } \quad \sigma_{\mathrm{H}_{n}, n}^{+} \leq t<\sigma_{0, n+1}^{-} \text {and } \sigma_{\mathrm{H}_{n}, n}^{+}<\infty, \\
& R(t)=R_{n+1}(t), \quad \text { if } \quad \sigma_{0, n+1}^{-} \leq t<\sigma_{\mathrm{H}_{n+1}, n+1}^{+} \text {and } \sigma_{0, n+1}^{-}<\infty .
\end{aligned}
$$

It can be verified that $\lim _{n \rightarrow \infty} \sigma_{0, n}^{-}=\lim _{n \rightarrow \infty} \sigma_{\mathrm{H}_{n}, n}^{+}=\infty$ almost surely under $\mathbb{P}_{x}$ with $x \in(0, \infty)$. Hence the process $R$ constructed as above is well defined.

For $a \in(0, \infty)$, let $\kappa_{a}^{+}:=\inf \{t \geq 0 ; R(t)>a\}$ be the first up-crossing time of level $a$ for the risk process $R$. In addition, for $a \in(0, \infty), x \in(0, a]$, we define

$$
f_{a}(x):=\mathbb{E}_{x}\left[\mathrm{e}^{-q \kappa_{a}^{+}}\right],
$$

and

$$
\begin{aligned}
g_{a}(x) & :=\mathbb{E}_{x}\left[\sum _ { n = 0 } ^ { \infty } \ell \left(\int_{\sigma_{\mathrm{H}_{n}, n}^{+}}^{\sigma_{0, n+1}^{-}} \mathrm{e}^{-q t} \mathrm{~d}\left(\sup _{\sigma_{\mathrm{H}_{n}, n}^{+} \leq s \leq t} X(s)-X\left(\sigma_{\mathrm{H}_{n}, n}^{+}\right)\right) \mathbf{1}_{\left\{\sigma_{0, n+1}^{-} \leq \kappa_{a}^{+}\right\}}\right.\right. \\
& \left.\left.+\int_{\sigma_{\mathrm{H}_{n}, n}^{+}}^{\kappa_{a}^{+}} \mathrm{e}^{-q t} \mathrm{~d}\left(\sup _{\sigma_{\mathrm{H}_{n}, n}^{+} \leq s \leq t} X(s)-X\left(\sigma_{\mathrm{H}_{n}, n}^{+}\right)\right) \mathbf{1}_{\left\{\sigma_{\mathrm{H}_{n}, n}^{+} \leq \kappa_{a}^{+}<\sigma_{0, n+1}^{-}\right\}}\right)\right],
\end{aligned}
$$

where $\sigma_{\mathrm{H}_{0}, 0}^{+}:=0$, and

$$
\begin{aligned}
r_{a}(x):= & \mathbb{E}_{x}\left[\sum_{n=1}^{\infty}\left(\mathrm{e}^{-q \sigma_{0, n}^{-}}\left|U_{n}\left(\sigma_{0, n}^{-}\right)\right|-\int_{\sigma_{0, n}^{-}}^{\sigma_{\mathrm{H}_{n}, n}^{+}} \mathrm{e}^{-q t} \mathrm{~d}\left(\inf _{s \in\left[\sigma_{0, n}^{-}, t\right]}\left(X(t)-X\left(\sigma_{0, n}^{-}\right)\right) \wedge 0\right)\right)\right. \\
& \left.\times \mathbf{1}_{\left\{\sigma_{\mathrm{H}_{n}, n}^{+} \leq \kappa_{a}^{+}\right\}}\right] .
\end{aligned}
$$

Hence, $g_{a}(x)$ represents the expected total discounted tax payments collected until the risk process $R$ reaches $a$, and $r_{a}(x)$ represents the expected total discounted capital injections made until the risk process $R$ reaches $a$.

The following result expresses the functions $f_{a}, g_{a}$ and $r_{a}$ by the scale functions $W_{q}$ and $Z_{q}$. It should be mentioned that the two identities in (11) can also be found in [6]. However, a new and transparent argument involving the excursion theory associated with Lévy processes is adopted in our case.

Proposition 3.1. For $q \geq 0, a \in(0, \infty)$, and $x \in(0, a]$, we have

$$
f_{a}(x)=\left(\frac{Z_{q}(x)}{Z_{q}(a)}\right)^{\frac{1}{1-\ell}}, \quad g_{a}(x)=\frac{\ell}{1-\ell} \int_{x}^{a}\left(\frac{Z_{q}(x)}{Z_{q}(w)}\right)^{\frac{1}{1-\ell}} \mathrm{d} w,
$$

and

$$
r_{a}(x)=\frac{1}{1-\ell} \int_{x}^{a}\left(Z_{q}(w)-\left(\bar{Z}_{q}(w)+\frac{\Psi^{\prime}(0+)}{q}\right) \frac{q W_{q}(w)}{Z_{q}(w)}\right)\left(\frac{Z_{q}(x)}{Z_{q}(w)}\right)^{\frac{1}{1-\ell}} \mathrm{d} w .
$$

Proof. The proof of this proporsition is given in Appendix B. 
By Proposition 3.1, the expected total discounted tax payments minus the expected total discounted costs of capital injection is given by

$$
\begin{aligned}
\bar{\psi}(x)= & g_{\infty}(x)-\varphi r_{\infty}(x) \\
= & -\frac{\varphi}{1-\ell} \int_{x}^{\infty}\left(Z_{q}(w)-\left(\bar{Z}_{q}(w)+\frac{\Psi^{\prime}(0+)}{q}\right) \frac{q W_{q}(w)}{Z_{q}(w)}\right)\left(\frac{Z_{q}(x)}{Z_{q}(w)}\right)^{\frac{1}{1-\ell}} \mathrm{d} w \\
& +\frac{\ell}{1-\ell} \int_{x}^{\infty}\left(\frac{Z_{q}(x)}{Z_{q}(w)}\right)^{\frac{1}{1-\ell}} \mathrm{d} w, \quad x \in(0, \infty),
\end{aligned}
$$

where $\varphi \in(1, \infty)$ is the cost per unit amount of capital injected. Define

$$
\bar{v}(x):=\bar{\psi}(x)-\varphi\left(\bar{Z}_{q}(x)+\frac{\Psi^{\prime}(0+)}{q}\right), \quad x \in(0, \infty) .
$$

If tax starts being collected only after the risk process reaches the level $a \in(0, \infty)$, then the expected total discounted delayed tax payments minus the expected total discounted cost of capital injection can be expressed as

$$
\begin{aligned}
\bar{\phi}(x ; a) & =\mathbb{E}_{x}\left[\mathrm{e}^{-q \rho_{a}^{+}}\right] \bar{\psi}(a)-\varphi \mathbb{E}_{x}\left[\int_{0}^{\rho_{a}^{+}} \mathrm{e}^{-q t} \mathrm{~d}\left(-\underline{X}_{t} \wedge 0\right)\right] \\
& =\frac{Z_{q}(x)}{Z_{q}(a)} \bar{\psi}(a)-\varphi \int_{x}^{a} \frac{Z_{q}(x)}{Z_{q}(w)}\left(Z_{q}(w)-\left(\bar{Z}_{q}(w)+\frac{\Psi^{\prime}(0+)}{q}\right) \frac{q W_{q}(w)}{Z_{q}(w)}\right) \mathrm{d} w \\
& =\frac{Z_{q}(x)}{Z_{q}(a)} \bar{\psi}(a)-\varphi\left(\left(\bar{Z}_{q}(a)+\frac{\Psi^{\prime}(0+)}{q}\right) \frac{Z_{q}(x)}{Z_{q}(a)}-\left(\bar{Z}_{q}(x)+\frac{\Psi^{\prime}(0+)}{q}\right)\right) \\
& =\frac{Z_{q}(x)}{Z_{q}(a)} \bar{v}(a)+\varphi\left(\bar{Z}_{q}(x)+\frac{\Psi^{\prime}(0+)}{q}\right)
\end{aligned}
$$

where we have used the Markov property, (19), and (12) with $\ell=0$.

For the convenience of presentation, we define two auxiliary functions as

$$
\bar{h}(a):=\bar{v}(a)-\bar{V}(a)\left(1-\varphi Z_{q}(a)\right) \quad \text { and } \quad \bar{V}(b):=\frac{Z_{q}(b)}{Z_{q}^{\prime}(b)} .
$$

The following result characterizes the global maximum point and the global maximum value of the function $a \mapsto \bar{\phi}(x ; a)$ for fixed $x$.

Theorem 3.1. Given $\phi \in(1, \infty)$. When $\bar{v}(0)>\bar{V}(0)\left(1-\varphi Z_{q}(0)\right)$, there is a unique positive solution $a^{+} \in(0, \infty)$ of the following equation

$$
\bar{h}(a)=0 \Leftrightarrow \bar{v}^{\prime}(a)=1-\varphi Z_{q}(a),
$$

and the function $\bar{\phi}(x ; a)$ (of a) attains its global maximum at $a^{*}=a^{+}$. Otherwise, when $\bar{v}(0) \leq$ $\bar{V}(0)\left(1-\varphi Z_{q}(0)\right),(16)$ has no positive solution, and the function $\bar{\phi}(x ; a)$ (of a) attains its global maximum at $a^{*}=0$.

Moreover, we have

$$
\bar{\phi}\left(x ; a^{*}\right)=\varphi\left(\bar{Z}_{q}(x)+\frac{\Psi^{\prime}(0+)}{q}\right)+\frac{Z_{q}(x)}{Z_{q}^{\prime}\left(a^{*}\right)}\left(1-\varphi Z_{q}\left(a^{*}\right)\right) .
$$


Proof. The proof of this theorem is given in Appendix B.

Remark 3.1. We would like to emphasize that, in Theorem 2.1 the characterization of the optimal tax implementation threshold $b^{*}$ relies on the assumption of the existence of a completely monotone density of the Lévy measure of $X$, whilst in Theorem 3.1, when capital injection is involved, no such assumption is needed to obtain the criterion of the optimal tax implementation threshold $a^{*}$.

A numerical example is given in Section 4 to illustrate the existence of positive optimal tax implementation threshold as given in Theorem 3.1 with discussions from the practical point of view.

\section{Numerical examples on optimal delay of taxation implementa- tion}

In this section we consider a Cramér-Lundberg risk model example. Let $X(t)=x+c t-S(t)$ with $x \geq 0$, and $S(t)$ is a compound Poisson process with rate $\lambda>0$ and an exponential jump distribution $F(x)=1-\mathrm{e}^{-\mu x}, \mu>0$. It can be verified that $X$ has a scale function

$$
W_{q}(x)=\frac{A_{1}(q)}{c} \mathrm{e}^{\theta_{1}(q) x}-\frac{A_{2}(q)}{c} \mathrm{e}^{\theta_{2}(q) x}, \quad x \geq 0, q \geq 0,
$$

where $\kappa(q)=\sqrt{(c \mu-\lambda-q)^{2}+4 c q \mu}, A_{1}(q)=\frac{\mu+\theta_{1}(q)}{\theta_{1}(q)-\theta_{2}(q)}=\frac{\lambda+q+c \mu}{2 \kappa(q)}+\frac{1}{2}, A_{2}(q)=\frac{\mu+\theta_{2}(q)}{\theta_{1}(q)-\theta_{2}(q)}=$ $\frac{\lambda+q+c \mu}{2 \kappa(q)}-\frac{1}{2}$, and

$$
\theta_{1}(q)=\frac{\lambda+q-c \mu+\kappa(q)}{2 c}, \quad \theta_{2}(q)=\frac{\lambda+q-c \mu-\kappa(q)}{2 c} .
$$

Further, for $x, q \geq 0$,

$$
\begin{aligned}
Z_{q}(x) & =1+q \int_{0}^{x}\left[\frac{A_{1}(q)}{c} \mathrm{e}^{\theta_{1}(q) y}-\frac{A_{2}(q)}{c} \mathrm{e}^{\theta_{2}(q) y}\right] \mathrm{d} y \\
& =\frac{q A_{1}(q)}{c \theta_{1}(q)} \mathrm{e}^{\theta_{1}(q) x}-\frac{q A_{2}(q)}{c \theta_{2}(q)} \mathrm{e}^{\theta_{2}(q) x} .
\end{aligned}
$$

Without loss of generality, we let $c=1.2, \mu=1, \lambda=1$, and $q=0.05$. In the following we shall present two numerical examples which illustrate the previously discussed two optimal delay of taxation implementation models respectively.

Example 1. We consider the optimal delay of taxation implementation with a terminal value at ruin first. For the purpose of comparison, we consider three levels of tax rate, i.e., $\ell=0.1,0.2$ and 0.3. To get a concrete idea on how the condition given in Theorem 2.1, i.e., $v(0)>V(0)(1-$ $\left.S q W_{q}(0)\right)$, behaves for varying $q$ and $S$ values, we show a $3 \mathrm{D}$ graph with $\ell=0.1, S \in[-5,+5]$ and $q \in(0,0.05]$ in Figure 1. It can be seen that when $q$ is extremely small $(q<0.004)$, the condition is met for certain ranges of positive $S$ values (the blue surface above the orange horizontal plain at $0)$. Otherwise, no positive terminal value can satisfy the condition of positive $b^{*}$. It implies that 
leaving a positive tax payment at the end cannot pair with taxation delay to achieve an maximal total expected discounted tax payments until ruin at normal rates of discounting.

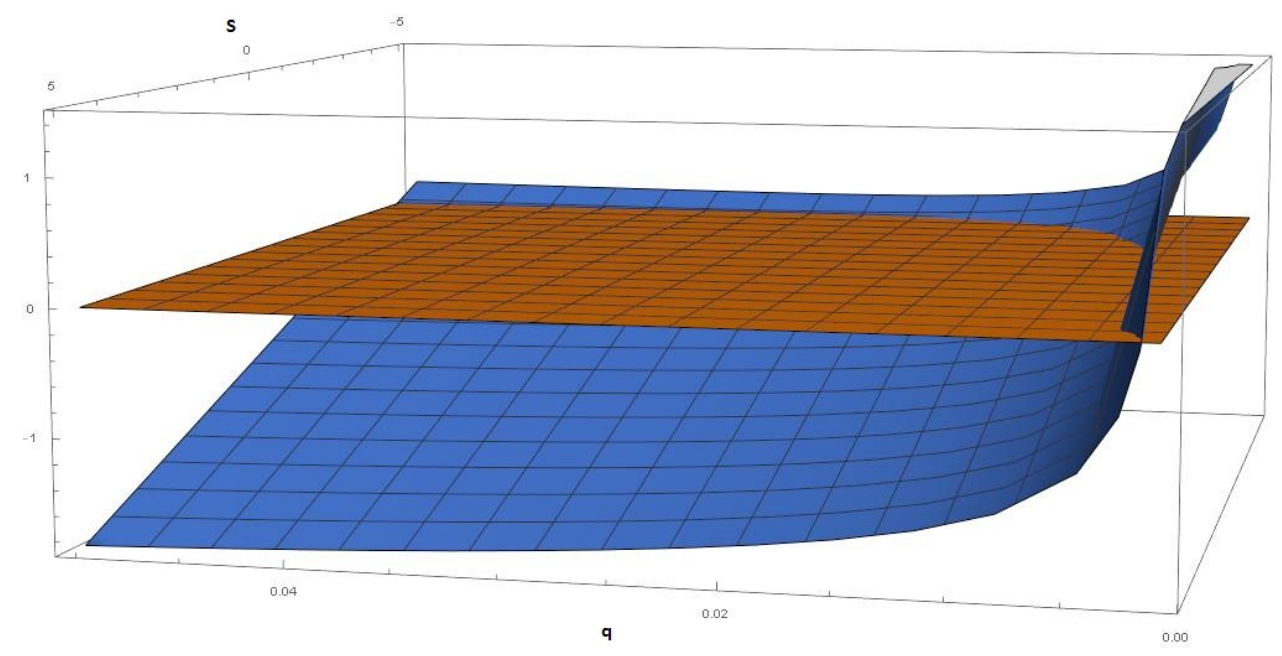

Figure 1: Optimal taxation implementation delay for various $S$ values

According to the condition of positive $b^{*}$ given in Theorem 2.1, we calculate the following ranges of $S$ values at each assumed $\ell$ level, which are summarized in Table 1 and Table 2. Further, Figure 2 and Figure 3 contain corresponding $b^{*}$ values for specifically chosen ranges for $S$. Table 1 and Figure 2 confirm the fact that it is worth implementing tax delays only when $S<0$ at $q=0.05$, i.e. the insurance company receives a tax benefit $S$ at ruin. From Table 2 and Figure 3 one can see that when $q=0.002$, both negative and certain ranged positive $S$ values lead to positive $b^{*}$ which means the delay in taxation implementation is worthwhile. In general, the optimal tax threshold level $b^{*}$ is a decreasing function of $S$. Also, given $S, b^{*}$ is an increasing function of $\ell$.

Table 1: Existence of positive $b^{*}$ at $q=0.05$

\begin{tabular}{|c|c|c|c|}
\hline$\ell$ & $v(0)$ & $V(0)\left(1-S q W_{q}(0)\right)$ & $b^{*}>0$ \\
\hline 0.1 & $0.29630-0.24801 S$ & $1.14286-0.04762 S$ & $S<-4.22$ \\
0.2 & $0.55487-0.21900 S$ & $1.14286-0.04762 S$ & $S<-3.43$ \\
0.3 & $0.77143-0.18931 S$ & $1.14286-0.04762 S$ & $S<-2.62$ \\
\hline
\end{tabular}


Table 2: Existence of positive $b^{*}$ at $q=0.002$

\begin{tabular}{|c|c|c|c|}
\hline$\ell$ & $v(0)$ & $V(0)\left(1-S q W_{q}(0)\right)$ & $b^{*}>0$ \\
\hline 0.1 & $1.75676-0.144583 S$ & $1.1976-0.001996 S$ & $S<3.92$ \\
0.2 & $2.87400-0.11451 S$ & $1.1976-0.001996 S$ & $S<14.9$ \\
0.3 & $3.36578-0.08521 S$ & $1.1976-0.001996 S$ & $S<26.06$ \\
\hline
\end{tabular}

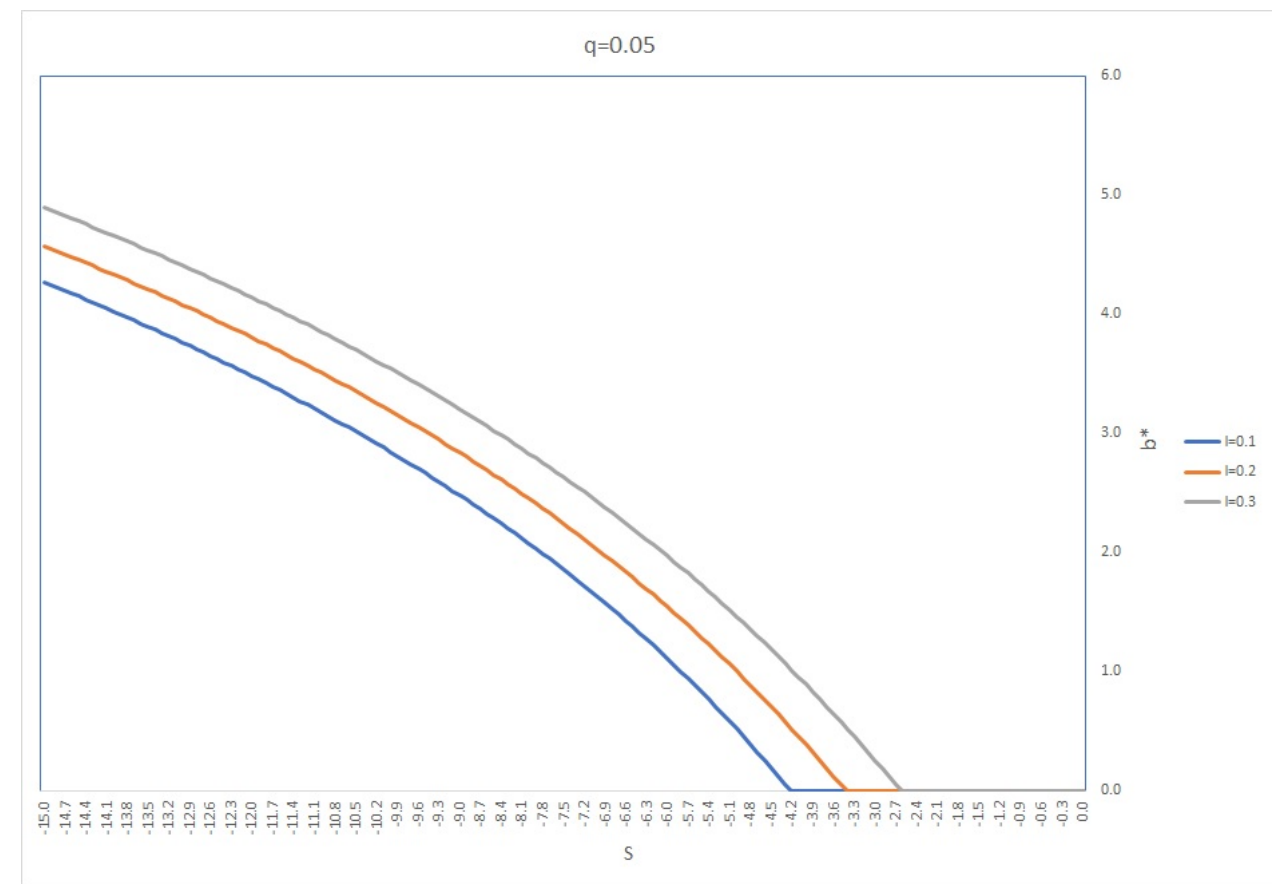

Figure 2: Optimal taxation implementation delay for various $S$ values 


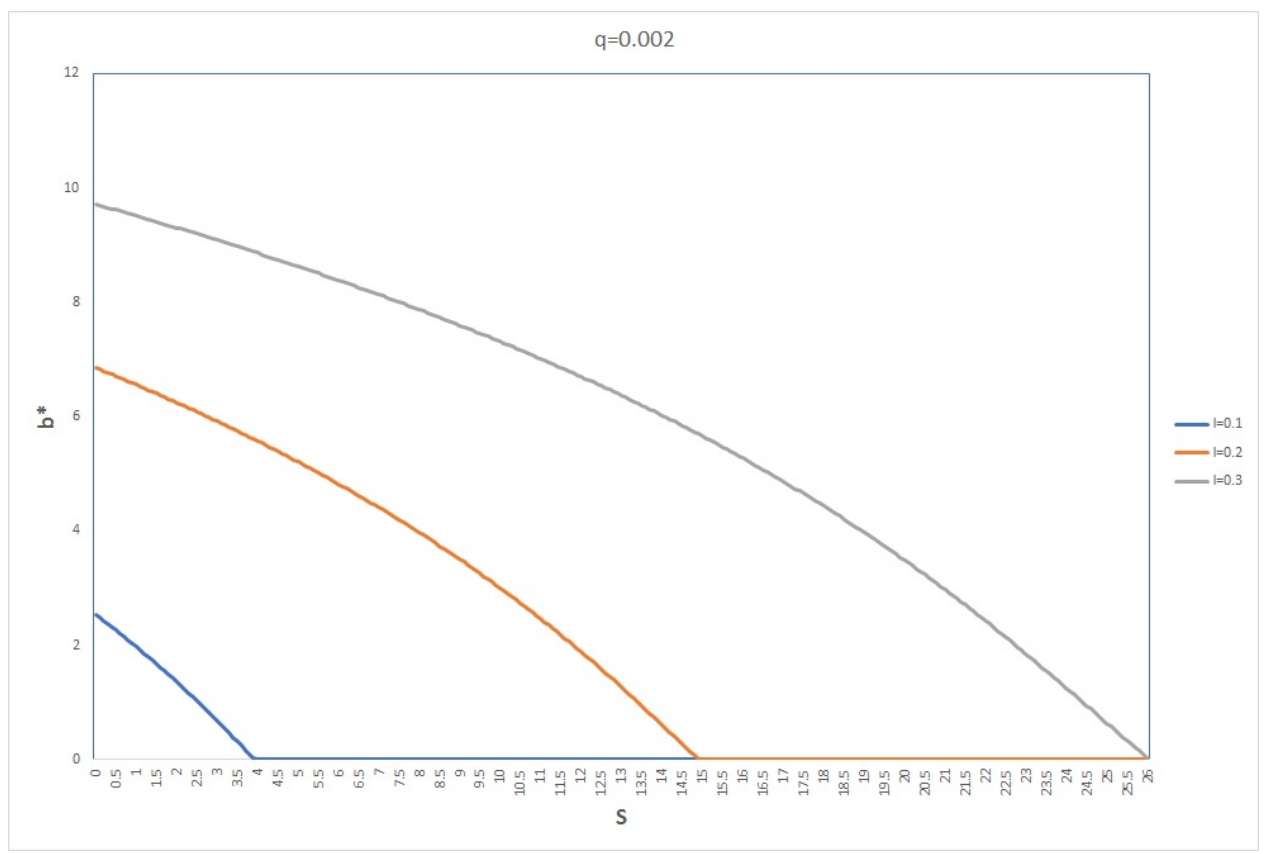

Figure 3: Optimal taxation implementation delay for various $S$ values

Remark 4.1. A negative terminal value of $S$, i.e., a benefit to the insurance company at ruin, can generally trigger a positive optimal tax implementation threshold level $b^{*}$, but a positive terminal value of $S$, eg a claw-back of early tax relief from the insurance company at ruin can only be effective when the discount rate is extremely small. It implies that in an extremely low interest rate environment, a terminal tax payment can also be used as an effective financial device to maximize the total discounted tax value.

Remark 4.2. The cause of claiming a tax benefit by the insurance company at ruin is due to the capital loss, which is obvious, according to the capital gain tax rule. The higher the initial tax implementation threshold is, the higher the potential tax benefit can be claimed at the end. It is consistent with our findings in Example 1.

Example 2. We consider the optimal delay of taxation implementation with capital injections in this example. Similarly, we shall consider three tax rates, $\ell=0.1,0.2$, and 0.3 , for comparison purpose. Let $q=0.05$. According to the condition of positive $a^{*}$ given in Theorem 3.1, we calculate the following ranges of $\varphi$ values at each assumed $\ell$ level, which are summarized in Table 3 . Further, Figure 4 shows the corresponding optimal taxation threshold levels for a specifically chosen range for $\varphi$. One can see from Table 3 and Figure 4 that it is worth implementing tax delays only when $\varphi>1$, which coincides with the definition of $\varphi$. The optimal tax threshold level $a^{*}$ is an increasing function of the cost of capital injection per dollar amount. Also, for the same cost of capital injection per dollar amount, a higher tax rate leads to a higher optimal tax threshold level. 
Table 3: Existence of positive $a^{*}$ in various cases

\begin{tabular}{|c|c|c|c|}
\hline$\ell$ & $\bar{v}(0)$ & $\bar{V}(0)\left(1-\varphi Z_{q}(0)\right)$ & $a^{*}>0$ \\
\hline 0.1 & $-2.9622-4 \varphi$ & $24-24 \varphi$ & $\varphi>1.348$ \\
0.2 & $-2.45091-4 \varphi$ & $24-24 \varphi$ & $\varphi>1.323$ \\
0.3 & $-1.93869-4 \varphi$ & $24-24 \varphi$ & $\varphi>1.297$ \\
\hline
\end{tabular}

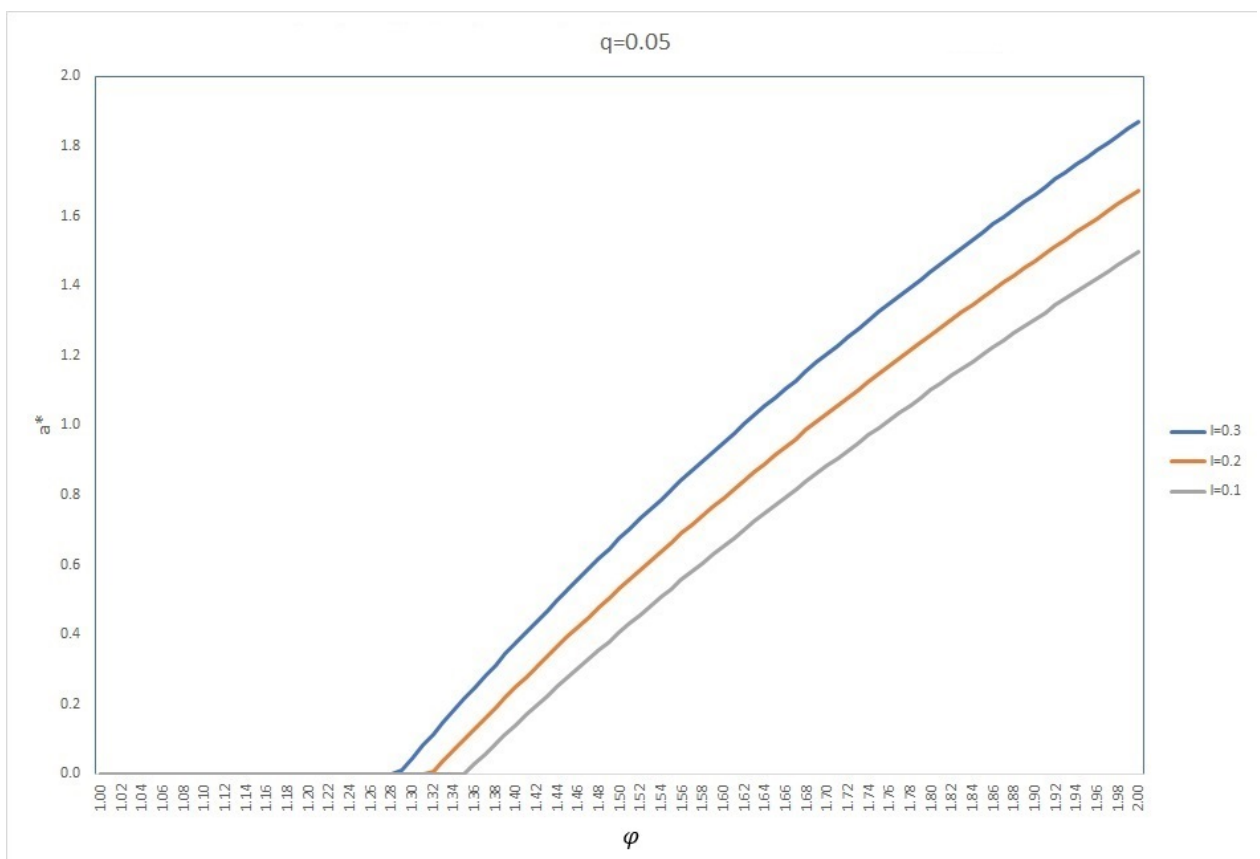

Figure 4: Optimal taxation implementation delay for various $\varphi$ values

Remark 4.3. The capital injection strategy seems more robust in respect of discount rates than the terminal value strategy. It is mainly because the capital injections could be needed at a reasonably early stage and the capital injections spread out the whole time line, whilst the terminal value can only occur at the very end, i.e. the time of ruin.

\section{Concluding remarks and future research}

This paper studied the optimal implementation delay of taxation problem within the spectrally negative Lévy insurance risk framework. Two types of trade-off were discussed when determining the optimal starting-tax surplus level: the trade-off between maximizing the total expected discounted tax payments until ruin and maximizing the expected discounted terminal value at ruin; the trade-off between maximizing the total expected discounted tax payments and minimizing the 
expected accumulated discounted costs of capital injection.

The consideration of the above mentioned two interesting trade-off problems on optimal implementation delay of loss-carry-forward taxation under the framework of the spectrally negative Lévy processes led to theoretical identification of the optimal starting-tax surplus levels as well as numerical insights of the potential behavior of the optimal starting-tax surplus level in various scenarios. One of the main observations made in this paper is that in general a terminal tax refund built in an assumed tax system helps to maximize the total discounted value of tax paid by an insurance company, whilst in an extremely low interest rate environment, a terminal tax payment can also be used as an effective financial device to maximize the total discounted tax value. It is evident that both terminal values and capital injections could facilitate the development of an optimal taxation system for insurance industries under the theoretically constructed economic environment. Although there is an obvious and significant gap between the scientific results and reality, we conclude that the results in this paper could shed a light for policymakers and insurance regulators on the following aspects.

First of all, insurance regulators and certain governance bodies play the key roles in regulating the insurance industry. The taxation authorities might not have direct involvements in this matter. This paper, on scientific aspect, showed that designing an optimal taxation system for the insurance industry can not only achieve the maximization of tax contributions from insurance companies, but also help to maintain the financial health of the whole insurance industry.

Secondly, taxation policymakers may only have a short-term focus when designing tax benefits for insurance industry, for instance, tax deductions due to capital losses can only date back a small number of years (3 or 5 years). The first numerical example in this paper showcased that both terminal tax refund and terminal tax payment could be effective financial devices to maximize the total discounted tax contributed by an insurance company when the whole lifespan of the company is in the picture.

This paper provides researchers in related fields with a good starting point for future research exploring the possibilities in narrowing the gap between theoretical modelling approaches and tax regulations in practice. Also, theoretical model extensions could also be considered on more structured 'terminal values', for instance, lump sum tax cash flows triggered by more general conditions (like down-crossing predetermined threshold level(s)).

\section{Acknowledgments}

The author Wenyuan Wang is very grateful to The University of Melbourne where part of the work on this paper was completed during his visit from July to September 2018. Wenyuan Wang is also supported in part by National Natural Science Foundation of China (No.11601197, No.11401498), Program for New Century Excellent Talents in Fujian Province University and NSERC (RGPIN2016-06704).

\section{References}

[1] Albrecher H, Avram F, Constantinescu C, Ivanovs J (2014a) The tax identity for Markov additive risk processes. Methodology and Computing in Applied Probability 16(1):245-258 
[2] Albrecher H, Badescu A, Landriault D (2008a) On the dual risk model with taxation. Insurance: Mathematics and Economics 42:1086-1094

[3] Albrecher H, Borst S, Boxma O, Resing J (2009) The tax identity in risk theory-a simple proof and an extension. Insurance: Mathematics and Economics 44:304-306

[4] Albrecher H, Borst S, Boxma O, \& Resing J (2011) Ruin excursions, the G/G/ $\infty$ queue, and tax payments in renewal risk models. Journal of Applied Probability 48(A):3-14

[5] Albrecher H, Hipp C (2007) Lundberg's risk process with tax. Blätter der DGVFM 28(1):1328

[6] Albrecher H, Ivanovs J (2014b) Power identities for Lévy risk models under taxation and capital injections. Stochastic Systems 4(1):157-172

[7] Albrecher H, Renaud J, Zhou X (2008b) A Lévy insurance risk process with tax. Journal of Applied Probability 45:363-375

[8] Avram F, Palmowski Z, Pistorius M (2007) On the optimal dividend problem for a spectrally negative Lévy process. The Annals of Applied Probability 17:156-180

[9] Avram F, Vu N, Zhou X (2017) On taxed spectrally negative Lévy processes with draw-down stopping. Insurance: Mathematics and Economics 76:69-74

[10] Bertoin J (1996) Lévy process. Cambridge University Press

[11] Chan T, Kyprianou A, Savov M (2011) Smoothness of scale functions for spectrally negative Lévy processes. Probability Theory and Related Fields 150 (3-4):691-708

[12] Cheung E, Landriault D (2012) On a risk model with surplus dependent premium and tax rates. Methodology and Computing in Applied Probability 14(2):233-251

[13] Cohen S, Kuznetsov A, Kyprianou A, Rivero V (2012) Lévy matters II: recent progress in theory and applications: fractional Lévy fields, and scale functions. Springer

[14] Feller, W., 1971. An introduction to probability theory and its applications. Vol. 2, 2nd edn. John Wiley, New York.

[15] Hao X, Tang Q (2009) Asymptotic ruin probabilities of the Lévy insurance model under periodic taxation. Astin Bulletin 39:479-494

[16] Kyprianou A (2006) Introductory lectures on fluctuations of Lévy processes with applications. Springer, Berlin

[17] Kyprianou A, Ott C (2012) Spectrally negative Lévy processes perturbed by functionals of their running supremum. Journal of Applied Probability 49(4):1005-1014

[18] Kyprianou A, Zhou X (2009) General tax structures and the Lévy insurance risk model. Journal of Applied Probability 46:1146-1156

[19] Li B, Tang Q, Zhou X (2013) A time-homogeneous diffusion model with tax. Journal of Applied Probability 50(1):195-207 
[20] Loeffen, R (2008) On optimality of the barrier strategy in de Finetti's dividend problem for spectrally negative Lévy processes. Annals of Applied Probability 18:1669-1680

[21] Loeffen R (2009) An optimal dividends problem with a terminal value for spectrally negative Lévy processes with a completely monotone jump density. Journal of Applied Probability 46(1):85-98

[22] Ming R, Wang W, Xiao L (2010) On the time value of absolute ruin with tax. Insurance: Mathematics and Economics 46:67-84

[23] Pistorius M (2004) On exit and ergodicity of the spectrally one-sided Lévy process reflected at its infimum. Journal of Theoretical Probability 17(1):183-220

[24] Renaud J (2009) The distribution of tax payments in a Lévy insurance risk model with a surplus-dependent taxation structure. Insurance: Mathematics and Economics 45:242-246

[25] Thonhauser S, Albrecher H (2007) Dividend maximization under consideration of the time value of ruin. Insurance: Mathematics and Economics 41(1):163-184

[26] Wang W, Chen P, Li S (2019) Generalized expected discounted penalty function at general drawdown for Lévy risk processes. https://arxiv.org/abs/1906.01449. Accessed 10 June 2019

[27] Wang W, Ming R, Hu Y (2011) On the expected discounted penalty function for risk process with tax. Statistics and Probability Letters 4:489-501

[28] Wang W, Hu Y (2012) Optimal loss-carry-forward taxation for the Lévy risk model. Insurance: Mathematics and Economics 50(1):121-130

[29] Wang W, Zhou X (2018) A draw-down reflected spectrally negative Lévy process. https://arxiv.org/abs/1812.06923. Accessed 15 May 2019

[30] Wang W, Zhou X (2018) General drawdown-based de Finetti optimization for spectrally negative Lévy risk processes. Journal of Applied Probability 55(2):513-542

[31] Wang S, Zhang C, Wang G (2010) A constant interest risk model with tax payments. Stochastic Models 26(3):384-398

[32] Wei J, Yang H, Wang R (2010) On the markov-modulated insurance risk model with tax. Blätter der DGVFM 31:65-78

[33] Wei L (2009) Ruin probability in the presence of interest earnings and tax payments. Insurance: Mathematics and Economics 45:133-138

[34] Zhang Z, Cheung E, Yang H (2017) Lévy insurance risk process with Poissonian taxation. Scandinavian Actuarial Journal 2017(1):51-87

[35] Zhou X (2007) Exit problems for spectrally negative Lévy processes reflected at either the supremum or the infimum. Journal of Applied Probability 44:1012-1030 


\section{Appendix A}

In this section, we briefly review some preliminaries of the spectrally negative Lévy process, namely a Lévy process with Lévy measure having support in $(-\infty, 0]$. Let $X=\{X(t) ; t \geq 0\}$ with probability laws $\left\{\mathbb{P}_{x} ; x \in \mathbb{R}\right\}$ and natural filtration $\left\{\mathcal{F}_{t} ; t \geq 0\right\}$ be a spectrally negative Lévy process, with the usual exclusion of pure increasing linear drift and the negative of a sub-ordinator. Denote by $\bar{X}$ and $\underline{X}$, i.e.

$$
\bar{X}(t)=\sup _{0 \leq s \leq t} X(s), \quad \underline{X}(t)=\inf _{0 \leq s \leq t} X(s), \quad t \geq 0,
$$

the running supremum and running infimum process of $X$, respectively.

Define the Laplace exponent of $X$ by

$$
\Psi(\theta):=\log \mathbb{E}_{x}\left[\mathrm{e}^{\theta(X(1)-x)}\right]=\gamma \theta+\frac{1}{2} \sigma^{2} \theta^{2}-\int_{(0, \infty)}\left(1-\mathrm{e}^{-\theta x}-\theta x \mathbf{1}_{(0,1)}(x)\right) \nu(\mathrm{d} x),
$$

where the Lévy measure $v$ satisfies $\int_{(0, \infty)}\left(1 \wedge x^{2}\right) \nu(\mathrm{d} x)<\infty$. It is known that $\Psi(\theta)$ is finite for $\theta \in[0, \infty)$ where it is strictly convex and infinitely differentiable. As defined in Theorem 8 on page 194 in [10], for each $q \geq 0$, the scale function $W_{q}:[0, \infty) \rightarrow[0, \infty)$ is the unique strictly increasing and continuous function with Laplace transform

$$
\int_{0}^{\infty} \mathrm{e}^{-\theta x} W_{q}(x) \mathrm{d} x=\frac{1}{\Psi(\theta)-q}, \quad \theta>\Phi(q)
$$

where $\Phi(q)$ is the largest solution of the equation $\Psi(\theta)=q$. For convenience, we extend the domain of $W_{q}$ to the whole real line by setting $W_{q}(x)=0$ for all $x<0$. In particular, write $W=W_{0}$ for simplicity. When $X$ has sample paths of unbounded variation, or when $X$ has sample paths of bounded variation and the Lévy measure has no atoms, the scale function $W_{q}$ is continuously differentiable over $(0, \infty)$. See [11] for more detailed discussions on the smoothness of scale functions.

We define

$$
Z_{q}(x)=1+q \int_{0}^{x} W_{q}(z) \mathrm{d} z \quad \text { and } \quad \bar{Z}_{q}(x)=\int_{0}^{x} Z_{q}(z) \mathrm{d} z, \quad q \geq 0, x \geq 0,
$$

with $Z_{q}(x)=1$ for $x<0$. For the process $X$, let its first down-crossing time of level 0 and first up-crossing time of level $b \in(0, \infty)$ be

$$
\tau_{0}^{-}:=\inf \{t \geq 0: X(t)<0\} \text { and } \tau_{b}^{+}:=\inf \{t \geq 0: X(t)>b\} .
$$

Then, Chapter 8 in [16] gives, for $q \geq 0$ and $b \in(0, \infty)$,

$$
\mathbb{E}_{x}\left[e^{-q \tau_{b}^{+}} \mathbf{1}_{\left\{\tau_{b}^{+}<\tau_{0}^{-}\right\}}\right]=\frac{W_{q}(x)}{W_{q}(b)}, \quad x \in(0, b] .
$$

Let $x \wedge y$ denote $\min (x, y)$ and let $x \vee y$ denote $\max (x, y)$. We now define a process $\{Y(t) ; t \geq 0\}$ as

$$
Y(t):=X(t)-\underline{X}(t) \wedge 0, \quad t \geq 0,
$$

which is the Lévy process $X$ reflected at its infimum. In risk theory, if $X_{t}$ denotes the surplus level of an insurance company at time $t$, then the term $-\underline{X}(t) \wedge 0$ could be used to represent the 
cumulative capital injection up to time $t$, and hence $Y_{t}$ is the surplus process with capital injections such that $Y(t) \geq 0$ for $t \geq 0$, i.e., ruin never occurs. Let

$$
\rho_{a}^{+}:=\inf \{t \geq 0 ; Y(t)>a\}, \quad a \in(0, \infty),
$$

which is the first up-crossing time of level $a$ for the risk process $Y$. Then, for $q \geq 0$ and $b \in(0, \infty)$, by Proposition 2 of [23], it holds that

$$
\mathrm{E}_{x}\left[\mathrm{e}^{-q \rho_{a}^{+}}\right]=Z_{q}(x) / Z_{q}(a), \quad x \in[0, a] .
$$

In addition, by the proof of Theorem 1 of [8] (see the 1st and 2nd blocks of equations on Page 167), we have

$$
\mathbb{E}\left[\int_{0}^{\rho_{a}^{+}} \mathrm{e}^{-q t} \mathrm{~d}(-\underline{X}(t) \wedge 0)\right]=-\frac{\Psi^{\prime}(0+)}{q}+\frac{\bar{Z}_{q}(a)+\frac{\Psi^{\prime}(0+)}{q}}{Z_{q}(a)}, \quad a \in(0, \infty),
$$

which is the expected total discounted capital injection made from time 0 until the risk process $Y$ hits $a$.

By Remark 4.5 of [30] and the proof of Theorem 3.10 in Page 136 of [13], we see that the function $\frac{W_{q}(x)}{W_{q}^{\prime}(x)}$ is non-decreasing with respect to $x$, i.e.,

$$
W_{q}(x) W_{q}^{\prime \prime}(x)<\left(W_{q}^{\prime}(x)\right)^{2}, \quad x \in(0, \infty) .
$$

From Proposition 2 (ii) of [23], one has

$$
\mathrm{E}_{0}\left[\mathrm{e}^{-q \bar{\tau}_{a}^{+}}\right]=Z_{q}(a)-\frac{q\left(W_{q}(a)\right)^{2}}{W_{q}^{\prime}(a)},
$$

where

$$
\bar{\tau}_{a}^{+}:=\inf \{t \geq 0 ; \bar{X}(s) \vee 0-X(t)>a\},
$$

is the first passage time of $X$ reflected at its supremum. Because $\lim _{a \rightarrow \infty} \bar{\tau}_{a}^{+}=\infty$, one knows

$$
\lim _{a \rightarrow \infty}\left(Z_{q}(a)-q \frac{\left(W_{q}(a)\right)^{2}}{W_{q}^{\prime}(a)}\right)=0
$$

In addition, it is found in [35] that

$$
\lim _{a \rightarrow \infty} \frac{W_{q}^{\prime}(a)}{W_{q}(a)}=\Phi(q)
$$

which together with the L'Hôpital's rule gives

$$
\lim _{a \rightarrow \infty} \frac{Z_{q}(a)}{Z_{q}^{\prime}(a)}=\frac{1}{\Phi(q)}
$$




\section{Appendix B}

\section{Theorem 2.1}

Proof. By (22), (23) and the L'Hôpital's rule, one has

$$
\lim _{b \rightarrow \infty} \int_{b}^{\infty}\left(\frac{W_{q}(b)}{W_{q}(z)}\right)^{\frac{1}{1-\ell}} \mathrm{d} z=\frac{1-\ell}{\Phi(q)}
$$

and

$$
\lim _{b \rightarrow \infty} \int_{b}^{\infty}\left(\frac{W_{q}(b)}{W_{q}(z)}\right)^{\frac{1}{1-\ell}}\left(\frac{W_{q}^{\prime}(z)}{W_{q}(z)} Z_{q}(z)-q W_{q}(z)\right) \mathrm{d} z=0 .
$$

Combining (23), (25) and (26) yields

$$
\begin{aligned}
& \lim _{b \rightarrow \infty}\left[v(b)-V(b)\left(1-S q W_{q}(b)\right)\right] \\
&=\lim _{b \rightarrow \infty}\left[\frac{S}{1-\ell} \int_{b}^{\infty}\left(\frac{W_{q}(b)}{W_{q}(z)}\right)^{\frac{1}{1-\ell}}\left(\frac{W_{q}^{\prime}(z)}{W_{q}(z)} Z_{q}(z)-q W_{q}(z)\right) \mathrm{d} z+S\left(q \frac{\left[W_{q}(b)\right]^{2}}{W_{q}^{\prime}(b)}-Z_{q}(b)\right)\right. \\
&\left.\quad+\frac{\ell}{1-\ell} \int_{b}^{\infty}\left(\frac{W_{q}(b)}{W_{q}(z)}\right)^{\frac{1}{1-\ell}} \mathrm{d} z-\frac{W_{q}(b)}{W_{q}^{\prime}(b)}\right] \\
&=\frac{\ell-1}{\Phi(q)}<0 .
\end{aligned}
$$

By $(8), \phi(x ; b)$ can be rewritten as

$$
\phi(x ; b)=S Z_{q}(x)+\frac{W_{q}(x)}{W_{q}(b)} v(b)
$$

from which one gets

$$
\begin{aligned}
\frac{\partial \phi(x ; b)}{\partial b} & =\frac{W_{q}(x)}{\left[W_{q}(b)\right]^{2}}\left[W_{q}(b) v^{\prime}(b)-W_{q}^{\prime}(b) v(b)\right] \\
& =\frac{\ell}{1-\ell} \frac{W_{q}(x) W_{q}^{\prime}(b)}{\left[W_{q}(b)\right]^{2}}\left[v(b)-V(b)\left(1-S Z_{q}^{\prime}(b)\right)\right],
\end{aligned}
$$

where the following expression for $v^{\prime}(b)$ is used to derive the second equality in (28)

$$
v^{\prime}(b)=\frac{1}{1-\ell} \frac{v(b)}{V(b)}+\frac{\ell}{1-\ell}\left(S Z_{q}^{\prime}(b)-1\right) .
$$

According to (28), $\frac{\partial \phi(x ; b)}{\partial b}=0$ is equivalent to (10), i.e., $b_{0}$ is a critical point of $b \mapsto \phi(x ; b)$ if $b_{0}$ solves (10). To specify the nature of this critical point, we examine the second partial derivative of $\phi(x ; b)$ with respect to $b$,

$$
\left.\frac{\partial^{2} \phi(x ; b)}{\partial b^{2}}\right|_{b=b_{0}}=\frac{\ell}{1-\ell} \frac{W_{q}(x) W_{q}^{\prime \prime}\left(b_{0}\right) v\left(b_{0}\right)}{\left[W_{q}\left(b_{0}\right)\right]^{2}}+\frac{\ell}{1-\ell} \frac{S q W_{q}(x) W_{q}^{\prime}\left(b_{0}\right)}{W_{q}\left(b_{0}\right)}
$$




$$
\begin{aligned}
& =\frac{\ell}{1-\ell} \frac{W_{q}(x)}{W_{q}\left(b_{0}\right)}\left(\frac{1-S q W_{q}\left(b_{0}\right)}{W_{q}^{\prime}\left(b_{0}\right)} W_{q}^{\prime \prime}\left(b_{0}\right)+S q W_{q}^{\prime}\left(b_{0}\right)\right) \\
& =\frac{\ell}{1-\ell} \frac{W_{q}(x)}{W_{q}\left(b_{0}\right)} f\left(b_{0}\right)
\end{aligned}
$$

where

$$
f(b):=\frac{1-S q W_{q}(b)}{W_{q}^{\prime}(b)} W_{q}^{\prime \prime}(b)+S q W_{q}^{\prime}(b) .
$$

In what follows, we are devoted to ruling out the possibility that the map $b \mapsto \phi(x ; b)$ has local minimum points or saddle points in $[0, \infty)$, by separately considering two opposite cases, i.e., Case A: $S \leq 0$ and Case B: $S>0$.

Case A. $S \leq 0$. In this case, we have $1-S q W_{q}(b)>0$ for all $b \in[0, \infty)$, and the following two claims A1 and A2 hold true.

A1. The map $b \mapsto \phi(x ; b)$ cannot have a local minimum point in $[0, \infty)$.

Indeed, by (27) and (28), there must exist a sufficiently large $\bar{b}>0$ such that $\phi(x ; b)$ is decreasing in $b$ over $[\bar{b}, \infty)$. Hence, if $b_{1}$ is a local minimum point of $b \mapsto \phi(x ; b)$, then there would have to exist a local maximum $b_{2} \in\left(b_{1}, \bar{b}\right]$, i.e.

$$
\left.\frac{\partial^{2} \phi(x ; b)}{\partial b^{2}}\right|_{b=b_{1}} \geq 0 \quad \text { and }\left.\quad \frac{\partial^{2} \phi(x ; b)}{\partial b^{2}}\right|_{b=b_{2}} \leq 0,
$$

or equivalently,

$$
f\left(b_{1}\right) \geq 0 \quad \text { and } \quad f\left(b_{2}\right) \leq 0 .
$$

However, as we assumed that the Lévy measure of $X$ has a completely monotone density, by Corollary 1 of [21], we know

$$
W_{q}^{\prime}(b) W_{q}^{\prime \prime \prime}(b)-\left(W_{q}^{\prime \prime}(b)\right)^{2}>0, \quad b \in(0, \infty),
$$

which gives

$$
f^{\prime}(b)=\left(1-S q W_{q}(b)\right) \frac{W_{q}^{\prime}(b) W_{q}^{\prime \prime \prime}(b)-\left[W_{q}^{\prime \prime}(b)\right]^{2}}{\left[W_{q}^{\prime}(b)\right]^{2}}>0, \quad b \in(0, \infty) .
$$

Clearly, (33) contradicts to (31), therefore claim A1 is true.

A2. The map $b \mapsto \phi(x ; b)$ cannot have a saddle point $b_{0}$ in $[0, \infty)$. Here, we say that $b_{0}$ is a saddle point of a function if both the first as well as the second derivative at $b_{0}$ of the function is 0 . 
Assume $b_{0}$ is such that

$$
v\left(b_{0}\right)=V\left(b_{0}\right)\left(1-S q W_{q}\left(b_{0}\right)\right) \quad \text { and }\left.\quad \frac{\partial^{2} \phi(x ; b)}{\partial b^{2}}\right|_{b=b_{0}}=0,
$$

one can verify that

$$
W_{q}^{\prime \prime}\left(b_{0}\right)=-\frac{S q\left[W_{q}^{\prime}\left(b_{0}\right)\right]^{2}}{1-S q W_{q}\left(b_{0}\right)} \quad \text { and } \quad V^{\prime}\left(b_{0}\right)=\frac{1}{1-S q W_{q}\left(b_{0}\right)} .
$$

For convenience, let

$$
h(b):=v(b)-V(b)\left(1-S q W_{q}(b)\right) .
$$

Clearly, $h\left(b_{0}\right)=0$. We will show that $b_{0}$ is a local minimum point of $h$. The first and second derivatives of $h$ are

$$
\begin{aligned}
h^{\prime}(b) & =v^{\prime}(b)-V^{\prime}(b)\left(1-S q W_{q}(b)\right)+S q W_{q}^{\prime}(b) V(b) \\
& =\frac{v(b)}{(1-\ell) V(b)}+\frac{\ell}{1-\ell}\left(S q W_{q}(b)-1\right)-\left(1-\frac{W_{q}(b) W_{q}^{\prime \prime}(b)}{\left[W_{q}^{\prime}(b)\right]^{2}}\right)\left(1-S q W_{q}(b)\right)+S q W_{q}(b) \\
& =\frac{h(b)}{(1-\ell)}+S q W_{q}(b)+\frac{W_{q}(b) W_{q}^{\prime \prime}(b)}{\left[W_{q}^{\prime}(b)\right]^{2}}\left(1-S q W_{q}(b)\right),
\end{aligned}
$$

and

$$
\begin{aligned}
h^{\prime \prime}(b)= & \frac{h^{\prime}(b)}{(1-\ell)}+S q W_{q}^{\prime}(b)-\frac{W_{q}(b) W_{q}^{\prime \prime}(b)}{\left[W_{q}^{\prime}(b)\right]^{2}} S q W_{q}^{\prime}(b) \\
& +\frac{\left(W_{q}^{\prime}(b) W_{q}^{\prime \prime}(b)+W_{q}(b) W_{q}^{\prime \prime \prime}(b)\right) W_{q}^{\prime}(b)-2 W_{q}(b)\left[W_{q}^{\prime \prime}(b)\right]^{2}}{\left[W_{q}^{\prime}(b)\right]^{3}}\left(1-S q W_{q}(b)\right) \\
= & \frac{h^{\prime}(b)}{(1-\ell)}+S q W_{q}^{\prime}(b)+\frac{W_{q}^{\prime \prime}(b)}{W_{q}^{\prime}(b)}\left(1-S q W_{q}(b)\right)+\frac{W_{q}(b) W_{q}^{\prime \prime \prime}(b)}{\left[W_{q}^{\prime}(b)\right]^{2}}\left(1-S q W_{q}(b)\right) \\
& -\frac{2 W_{q}(b)\left[W_{q}^{\prime \prime}(b)\right]^{2}}{\left[W_{q}^{\prime}(b)\right]^{3}}\left(1-S q W_{q}(b)\right)-\frac{S q W_{q}(b) W_{q}^{\prime \prime}(b)}{W_{q}^{\prime}(b)} .
\end{aligned}
$$

One can see that $h^{\prime}\left(b_{0}\right)=0$, and

$$
S q W_{q}^{\prime}\left(b_{0}\right)+\frac{W_{q}^{\prime \prime}\left(b_{0}\right)}{W_{q}^{\prime}\left(b_{0}\right)}\left(1-S q W_{q}\left(b_{0}\right)\right)=0 .
$$

Also, we have

$$
-\frac{2 W_{q}\left(b_{0}\right)\left[W_{q}^{\prime \prime}\left(b_{0}\right)\right]^{2}}{\left[W_{q}^{\prime}\left(b_{0}\right)\right]^{3}}\left(1-S q W_{q}\left(b_{0}\right)\right)-\frac{S q W_{q}\left(b_{0}\right) W_{q}^{\prime \prime}\left(b_{0}\right)}{W_{q}^{\prime}\left(b_{0}\right)}=-\frac{S^{2} q^{2} W_{q}\left(b_{0}\right) W_{q}^{\prime}\left(b_{0}\right)}{1-S q W_{q}\left(b_{0}\right)} .
$$

By (32) we obtain

$$
h^{\prime \prime}\left(b_{0}\right)=\frac{W_{q}\left(b_{0}\right) W_{q}^{\prime \prime \prime}\left(b_{0}\right)}{\left[W_{q}^{\prime}\left(b_{0}\right)\right]^{2}}\left(1-S q W_{q}\left(b_{0}\right)\right)-\frac{S^{2} q^{2} W_{q}\left(b_{0}\right) W_{q}^{\prime}\left(b_{0}\right)}{1-S q W_{q}\left(b_{0}\right)}
$$




$$
\begin{aligned}
& >\frac{W_{q}\left(b_{0}\right)\left[W_{q}^{\prime \prime}\left(b_{0}\right)\right]^{2}}{\left[W_{q}^{\prime}\left(b_{0}\right)\right]^{3}}\left(1-S q W_{q}\left(b_{0}\right)\right)-\frac{S^{2} q^{2} W_{q}\left(b_{0}\right) W_{q}^{\prime}\left(b_{0}\right)}{1-S q W_{q}\left(b_{0}\right)} \\
& =\frac{W_{q}\left(b_{0}\right)\left(1-S q W_{q}\left(b_{0}\right)\right)}{\left[W_{q}^{\prime}\left(b_{0}\right)\right]^{3}}\left[\left(W_{q}^{\prime \prime}\left(b_{0}\right)\right)^{2}-\frac{S^{2} q^{2}\left(W_{q}^{\prime}\left(b_{0}\right)\right)^{4}}{\left(1-S q W_{q}\left(b_{0}\right)\right)^{2}}\right]=0 .
\end{aligned}
$$

As a result, $h(b)$ reaches a local minimum value of 0 at $b_{0}$. Hence, $h(b)>0$ holds true for all $b \in\left(b_{0}, b_{0}+\epsilon_{0}\right)$ for some $\epsilon_{0}>0$, which combined with (28) implies $\frac{\partial \phi(x ; b)}{\partial b}>0$ for all $b \in\left(b_{0}, b_{0}+\epsilon_{0}\right)$. Therefore, the saddle point $b_{0}$ of the map $b \mapsto \phi(x ; b)$, has to be followed by a maximum or another saddle point $b_{1} \in\left(b_{0}, \infty\right)$ of the map $b \mapsto \phi(x ; b)$, i.e., $f\left(b_{0}\right)=0$ and $f\left(b_{1}\right) \leq 0$ (c.f., (29) and (30)), contradicting to (33). Hence the claim A2 is true.

Case B. $S>0$. The following two claims B1 and B2 are true.

B1. The map $b \mapsto \phi(x ; b)$ cannot have a local minimum in $[0, \infty)$.

If there exists a local minimum point $b_{1}$ of the map $b \mapsto \phi(x ; b)$, using $(27)$ and $(28)$ once again we can see that there exists a local maximum point $b_{2} \in\left(b_{1}, \infty\right)$, and then we obtain (c.f., (29) and (30))

$$
f\left(b_{1}\right)>0 \text { and } f\left(b_{2}\right)<0 .
$$

Combining (30) with (21) yields

$$
\begin{aligned}
f(b) & =\frac{1-S q W_{q}(b)}{W_{q}^{\prime}(b)} W_{q}^{\prime \prime}(b)+S q W_{q}^{\prime}(b) \\
& =\frac{W_{q}^{\prime \prime}(b)}{W_{q}^{\prime}(b)}-\frac{S q W_{q}(b) W_{q}^{\prime \prime}(b)}{W_{q}^{\prime}(b)}+S q W_{q}^{\prime}(b)>\frac{W_{q}^{\prime \prime}(b)}{W_{q}^{\prime}(b)},
\end{aligned}
$$

which, together with (34), implies that

$$
W_{q}^{\prime \prime}\left(b_{2}\right)<0
$$

- If $1-S q W_{q}\left(b_{2}\right) \geq 0$, the strict increasing property of the scale function $W_{q}(b)$ gives $1-S q W_{q}(b) \geq 0$ for $b \in\left[b_{1}, b_{2}\right]$. Similar to the derivation of result (33), we can show that $f^{\prime}(b)>0$ for $b \in\left[b_{1}, b_{2}\right]$, which contradicts to the result of $f\left(b_{2}\right)<0$ given in (34).

- Otherwise, if $1-S q W_{q}\left(b_{2}\right)<0$, from $f\left(b_{2}\right)=\frac{1-S q W_{q}\left(b_{2}\right)}{W_{q}^{\prime}\left(b_{2}\right)} W_{q}^{\prime \prime}\left(b_{2}\right)+S q W_{q}^{\prime}\left(b_{2}\right)<0$, it holds that $W_{q}^{\prime \prime}\left(b_{2}\right)>\frac{-S q\left[W_{q}^{\prime}\left(b_{2}\right)\right]^{2}}{1-S q W_{q}\left(b_{2}\right)}>0$, which contradicts to $(35)$.

Therefore, the claim B1 is true. 
B2. The map $b \mapsto \phi(x ; b)$ cannot have a saddle point $b_{0}$ in $[0, \infty)$.

Assume that $b_{0}$ is a saddle point in $[0, \infty)$.

- If $1-S q W_{q}\left(b_{0}\right)>0$, adopting the same arguments as in the claim $\mathbf{A}_{2}$, we can show that $h(b)$ reaches a local minimum value of 0 at $b_{0}$, which, together with (27) and (28), implies that there must be a local maximum point or another saddle point $b_{1} \in\left(b_{0}, \infty\right)$ of the map $b \mapsto \phi(x ; b)$, i.e.

$$
f\left(b_{1}\right) \leq 0=f\left(b_{0}\right)
$$

If $1-S q W_{q}\left(b_{1}\right) \geq 0,(33)$ holds true for all $b \in\left[b_{0}, b_{1}\right)$, contradicting to (36). Otherwise, if $1-S q W_{q}\left(b_{1}\right)<0$, by $\frac{1-S q W_{q}\left(b_{1}\right)}{W_{q}^{\prime}\left(b_{1}\right)} W_{q}^{\prime \prime}\left(b_{1}\right)+S q W_{q}^{\prime}\left(b_{1}\right)=f\left(b_{1}\right) \leq 0$ we get $W_{q}^{\prime \prime}\left(b_{1}\right) \geq$ $\frac{-S q\left[W_{q}^{\prime}\left(b_{1}\right)\right]^{2}}{1-S q W_{q}\left(b_{1}\right)}>0$, contradicting to $W_{q}^{\prime \prime}\left(b_{1}\right) \leq 0$ (c.f., arguments used in verifying $\left.(35)\right)$.

- If $1-S q W_{q}\left(b_{0}\right) \leq 0$, adopting the same arguments as in verifying (35) we have $W_{q}^{\prime \prime}\left(b_{0}\right)<$ 0 . However, by $f\left(b_{0}\right)=0$, one can see that $W_{q}^{\prime \prime}\left(b_{0}\right)=-\frac{S q\left[W_{q}^{\prime}\left(b_{0}\right)\right]^{2}}{1-S q W_{q}\left(b_{0}\right)}>0$, which is a contradiction.

Hence, the claim B2 is true.

Summing up the arguments concerning Case A and Case B, we conclude that (10) has at most one positive solution.

- When $v(0)>V(0)\left(1-S q W_{q}(0)\right)$, by $(27)$, one concludes that a positive solution of (10), denoted by $b^{+} \in(0, \infty)$, exists and satisfies

$$
v(b)>V(b)\left(1-S q W_{q}(b)\right), b \in\left[0, b^{+}\right) \quad \text { and } \quad v(b)<V(b)\left(1-S q W_{q}(b)\right), b \in\left(b^{+}, \infty\right),
$$

i.e., the map $b \mapsto \phi(x ; b)$ reaches its global maximum value at $b^{*}=b^{+}$.

- When $v(0)<V(0)\left(1-S q W_{q}(0)\right)$, the equation (10) has no positive solution and

$$
v(b)<V(b)\left(1-S q W_{q}(b)\right), b \in[0, \infty),
$$

i.e., the map $b \mapsto \phi(x ; b)$ reaches its global maximum value at $b^{*}=0$.

- When $v(0)=V(0)\left(1-S q W_{q}(0)\right)$, then (37) holds true for $b \in(0, \infty)$ and the map $b \mapsto \phi(x ; b)$ reaches its global maximum value at $b^{*}=0$.

The proof of Theorem 2.1 is completed. 


\section{Proporsition 3.1}

Proof. From (2), (5) and the Markov property, it is seen that, for $a \in(0, \infty), x \in(0, a]$,

$$
\begin{aligned}
f_{a}(x) & =\mathbb{E}_{x}\left[\mathrm{e}^{-q \sigma_{a}^{+}} ; \sigma_{a}^{+}<\sigma_{0}^{-}\right]+\mathbb{E}_{x}\left[\mathrm{e}^{-q \sigma_{0}^{-}}\left(\left.\mathbb{E}_{0}\left[\mathrm{e}^{-q \rho \rho_{w}^{+}}\right]\right|_{w=\mathrm{H}_{1}}\right) \mathbb{E}_{\mathrm{H}_{1}}\left[\mathrm{e}^{-q \kappa_{a}^{+}}\right] ; \sigma_{0}^{-}<\sigma_{a}^{+}\right] \\
& =\left(\frac{W_{q}(x)}{W_{q}(a)}\right)^{\frac{1}{1-\ell}}+\mathbb{E}_{x}\left[\mathrm{e}^{-q \sigma_{0}^{-}} \frac{f_{a}\left(\mathrm{H}_{1}\right)}{Z_{q}\left(\mathrm{H}_{1}\right)} ; \sigma_{0}^{-}<\sigma_{a}^{+}\right] \\
& =\left(\frac{W_{q}(x)}{W_{q}(a)}\right)^{\frac{1}{1-\ell}}+\frac{1}{1-\ell} \int_{x}^{a} \frac{f_{a}(s)}{Z_{q}(s)}\left(\frac{W_{q}(x)}{W_{q}(s)}\right)^{\frac{1}{1-\ell}}\left(\frac{W_{q}^{\prime}(s)}{W_{q}(s)} Z_{q}(s)-q W_{q}(s)\right) \mathrm{d} s .
\end{aligned}
$$

Differentiating both sides of (38) with respect to $x$ gives the following differential equation, for $x \in(0, \infty)$,

$$
\begin{aligned}
f_{a}^{\prime}(x)= & \frac{1}{1-\ell} \frac{W_{q}^{\prime}(x)}{W_{q}(x)}\left(\frac{W_{q}(x)}{W_{q}(a)}\right)^{\frac{1}{1-\ell}}+\frac{1}{1-\ell} \frac{W_{q}^{\prime}(x)}{W_{q}(x)}\left(f_{a}(x)-\left(\frac{W_{q}(x)}{W_{q}(a)}\right)^{\frac{1}{1-\ell}}\right) \\
& -\frac{f_{a}(x)}{1-\ell}\left(\frac{W_{q}^{\prime}(x)}{W_{q}(x)}-\frac{q W_{q}(x)}{Z_{q}(x)}\right) \\
= & \frac{1}{1-\ell} \frac{q W_{q}(x)}{Z_{q}(x)} f_{a}(x)
\end{aligned}
$$

with boundary condition $f_{a}(a)=1$. Solving (39), we obtain the first identity in (11). By (4) and an argument similar to the one used in deriving (38), we have

$$
\begin{aligned}
g_{a}(x)= & \mathbb{E}_{x}\left[\int_{0}^{\sigma_{a}^{+} \wedge \sigma_{0}^{-}} \mathrm{e}^{-q t} \ell \mathrm{d} \bar{X}(t)\right]+\mathbb{E}_{x}\left[\mathrm{e}^{-q \sigma_{0}^{-}} \frac{g\left(\mathrm{H}_{1}\right)}{Z_{q}\left(\mathrm{H}_{1}\right)} ; \sigma_{0}^{-}<\sigma_{a}^{+}\right] \\
= & \frac{\ell}{1-\ell} \int_{x}^{a}\left(\frac{W_{q}(x)}{W_{q}(s)}\right)^{\frac{1}{1-\ell}} \mathrm{d} s+\frac{1}{1-\ell} \int_{x}^{a} \frac{g(s)}{Z_{q}(s)}\left(\frac{W_{q}(x)}{W_{q}(s)}\right)^{\frac{1}{1-\ell}} \\
& \times\left(\frac{W_{q}^{\prime}(s)}{W_{q}(s)} Z_{q}(s)-q W_{q}(s)\right) \mathrm{d} s, \quad x \in(0, \infty), \quad a \in(x, \infty) .
\end{aligned}
$$

Differentiating both sides of (40) with respect to $x$ gives the following differential equation

$$
\begin{aligned}
g_{a}^{\prime}(x) & =\frac{1}{1-\ell} \frac{W_{q}^{\prime}(x)}{W_{q}(x)} g(x)-\frac{\ell}{1-\ell}-\frac{g(x)}{1-\ell}\left(\frac{W_{q}^{\prime}(x)}{W_{q}(x)}-\frac{q W_{q}(x)}{Z_{q}(x)}\right) \\
& =\frac{1}{1-\ell} \frac{q W_{q}(x)}{Z_{q}(x)} g(x)-\frac{\ell}{1-\ell}, \quad x \in(0, \infty),
\end{aligned}
$$

with boundary condition $g_{a}(a)=0$. Solving (41) gives the second identity in (11).

Combining (20), (5) and (6) yields, for $a \in(0, \infty)$,

$$
\left.r_{a}(x)=\mathbb{E}_{x}\left[\mathrm{e}^{-q \sigma_{0}^{-}}\left|U\left(\sigma_{0}^{-}\right)\right| ; \sigma_{0}^{-}<\sigma_{a}^{+}\right]+\mathbb{E}_{x}\left[\mathrm{e}^{-q \sigma_{0}^{-}} \mathbb{E}\left[\int_{0}^{\rho_{\mathrm{H}_{1}}^{+}} \mathrm{e}^{-q t} \mathrm{~d}(-\underline{X}(t) \wedge 0)\right)\right] ; \sigma_{0}^{-}<\sigma_{a}^{+}\right]
$$




$$
\begin{aligned}
& +\mathbb{E}_{x}\left[\mathrm{e}^{-q \sigma_{0}^{-}} \frac{r_{a}\left(\mathrm{H}_{1}\right)}{Z_{q}\left(\mathrm{H}_{1}\right)} ; \sigma_{0}^{-}<\sigma_{a}^{+}\right] \\
= & \frac{1}{1-\ell} \int_{x}^{a}\left(\frac{W_{q}(x)}{W_{q}(s)}\right)^{\frac{1}{1-\ell}}\left(Z_{q}(s)-\Psi^{\prime}(0+) W_{q}(s)-\frac{\bar{Z}_{q}(s)-\Psi^{\prime}(0+) \bar{W}_{q}(s)}{W_{q}(s)} W_{q}^{\prime}(s)\right) \mathrm{d} s \\
& +\frac{1}{1-\ell} \int_{x}^{a}\left(\frac{W_{q}(x)}{W_{q}(s)}\right)^{\frac{1}{1-\ell}}\left(-\frac{\Psi^{\prime}(0+)}{q}+\frac{\bar{Z}_{q}(s)+\frac{\Psi^{\prime}(0+)}{q}}{Z_{q}(s)}+\frac{r_{a}(s)}{Z_{q}(s)}\right) \\
& \times\left(\frac{W_{q}^{\prime}(s)}{W_{q}(s)} Z_{q}(s)-q W_{q}(s)\right) \mathrm{d} s, \quad x \in(0, a] .
\end{aligned}
$$

Differentiating both sides of (42) with respect to $x$ gives the following differential equation

$$
\begin{aligned}
r_{a}^{\prime}(x)= & \frac{1}{1-\ell} \frac{W_{q}^{\prime}(x)}{W_{q}(x)} r_{a}(x)-\frac{1}{1-\ell}\left(Z_{q}(x)-\Psi^{\prime}(0+) W_{q}(x)-\frac{\bar{Z}_{q}(x)-\Psi^{\prime}(0+) \bar{W}_{q}(x)}{W_{q}(x)} W_{q}^{\prime}(x)\right) \\
& -\frac{1}{1-\ell}\left(-\frac{\Psi^{\prime}(0+)}{q}+\frac{\bar{Z}_{q}(x)+\frac{\Psi^{\prime}(0+)}{q}}{Z_{q}(x)}+\frac{r_{a}(x)}{Z_{q}(x)}\right)\left(\frac{W_{q}^{\prime}(x)}{W_{q}(x)} Z_{q}(x)-q W_{q}(x)\right) \\
= & \frac{1}{1-\ell} \frac{q W_{q}(x)}{Z_{q}(x)} r_{a}(x)-\frac{1}{1-\ell}\left(Z_{q}(x)-\left(\bar{Z}_{q}(x)+\frac{\Psi^{\prime}(0+)}{q}\right) \frac{q W_{q}(x)}{Z_{q}(x)}\right), \quad x \in(0, a],
\end{aligned}
$$

with boundary condition $r_{a}(a)=0$. Solving (43) gives (12). The proof is completed.

\section{Theorem 3.1}

Proof. Firstly, we show a result that is useful in our main proof:

$$
\lim _{a \rightarrow \infty} \bar{h}(a)=\frac{\ell-1}{\Phi(q)}<0
$$

Using integration by parts, one can get

$$
\begin{aligned}
& -\frac{1}{1-\ell} \int_{a}^{\infty}\left(\bar{Z}_{q}(w)+\frac{\Psi^{\prime}(0+)}{q}\right) \frac{q W_{q}(w)}{Z_{q}(w)}\left(\frac{Z_{q}(a)}{Z_{q}(w)}\right)^{\frac{1}{1-\ell}} \mathrm{d} w \\
= & \left.\left(\bar{Z}_{q}(w)+\frac{\Psi^{\prime}(0+)}{q}\right)\left(\frac{Z_{q}(a)}{Z_{q}(w)}\right)^{\frac{1}{1-\ell}}\right|_{w=a} ^{\infty}-\int_{a}^{\infty} Z_{q}(w)\left(\frac{Z_{q}(a)}{Z_{q}(w)}\right)^{\frac{1}{1-\ell}} \mathrm{d} w \\
= & -\bar{Z}_{q}(a)-\frac{\Psi^{\prime}(0+)}{q}-\int_{a}^{\infty} Z_{q}(w)\left(\frac{Z_{q}(a)}{Z_{q}(w)}\right)^{\frac{1}{1-\ell}} \mathrm{d} w, \quad a \in(0, \infty),
\end{aligned}
$$

and

$$
\begin{aligned}
& -\frac{\ell}{1-\ell} \int_{a}^{\infty} Z_{q}(w)\left(\frac{Z_{q}(a)}{Z_{q}(w)}\right)^{\frac{1}{1-\ell}} \mathrm{d} w \\
= & \int_{a}^{\infty} \frac{Z_{q}(w)\left(Z_{q}(a)\right)^{\frac{1}{1-\ell}}}{Z_{q}^{\prime}(w)} \mathrm{d}\left(Z_{q}(w)\right)^{-\frac{1}{1-\ell}+1}
\end{aligned}
$$




$$
=-\bar{V}(a) Z_{q}(a)-\int_{a}^{\infty} Z_{q}(w)\left(\frac{Z_{q}(w)}{Z_{q}^{\prime}(w)}\right)^{\prime}\left(\frac{Z_{q}(a)}{Z_{q}(w)}\right)^{\frac{1}{1-\ell}} \mathrm{d} w, \quad a \in(0, \infty) .
$$

Hence, by (13) and (15) we have

$$
\begin{aligned}
\bar{h}(a)= & \frac{\ell}{1-\ell} \int_{a}^{\infty}\left(\frac{Z_{q}(a)}{Z_{q}(w)}\right)^{\frac{1}{1-\ell}} \mathrm{d} w-\frac{Z_{q}(a)}{Z_{q}^{\prime}(a)} \\
& -\varphi \int_{a}^{\infty} Z_{q}(w) \frac{q\left(W_{q}(w)\right)^{2} / W_{q}^{\prime}(w)-Z_{q}(w)}{q\left(W_{q}(w)\right)^{2} / W_{q}^{\prime}(w)}\left(\frac{Z_{q}(a)}{Z_{q}(w)}\right)^{\frac{1}{1-\ell}} \mathrm{d} w .
\end{aligned}
$$

Using (22), (24) and L'Hôpital's rule, we get

$$
\lim _{a \rightarrow \infty} \frac{\ell}{1-\ell} \int_{a}^{\infty}\left(\frac{Z_{q}(a)}{Z_{q}(w)}\right)^{\frac{1}{1-\ell}} \mathrm{d} w=\frac{\ell}{\Phi(q)}
$$

and

$$
\lim _{a \rightarrow \infty} \int_{a}^{\infty} Z_{q}(w) \frac{q\left(W_{q}(w)\right)^{2} / W_{q}^{\prime}(w)-Z_{q}(w)}{q\left(W_{q}(w)\right)^{2} / W_{q}^{\prime}(w)}\left(\frac{Z_{q}(a)}{Z_{q}(w)}\right)^{\frac{1}{1-\ell}} \mathrm{d} w=0,
$$

which, together with (24) and (45), gives (44).

Then, we show that the function $\bar{h}(a)$ can be used to find critical points of $a \mapsto \bar{\phi}(x ; a)$. By the expression of $\bar{\phi}(x ; a)$ given by $(14)$, we have

$$
\begin{aligned}
\frac{\partial \bar{\phi}(x ; a)}{\partial a} & =\frac{Z_{q}(x)}{\left(Z_{q}(a)\right)^{2}}\left(Z_{q}(a) \bar{v}^{\prime}(a)-Z_{q}^{\prime}(a) \bar{v}(a)\right) \\
& =\frac{\ell}{1-\ell} \frac{Z_{q}(x) Z_{q}^{\prime}(a)}{\left(Z_{q}(a)\right)^{2}}\left[\bar{v}(a)-\bar{V}(a)\left(1-\varphi Z_{q}(a)\right)\right] \\
& =\frac{\ell}{1-\ell} \frac{Z_{q}(x) Z_{q}^{\prime}(a)}{\left(Z_{q}(a)\right)^{2}} \bar{h}(a)
\end{aligned}
$$

where the following expression for $\bar{v}^{\prime}(a)$ is employed

$$
\bar{v}^{\prime}(a)=\frac{1}{1-\ell} \frac{\bar{v}(a)}{\bar{V}(a)}+\frac{\ell}{1-\ell}\left(\varphi Z_{q}(a)-1\right) .
$$

According to (46), $\frac{\partial \bar{\phi}(x ; a)}{\partial a}=0$ is equivalent to (16), i.e., $a_{0}$ is a critical point of $a \mapsto \bar{\phi}(x ; a)$ if $a_{0}$ solves (16). To specify the nature of this critical point $a_{0}$, we examine the second partial derivative of $\bar{\phi}(x ; a)$,

$$
\begin{aligned}
\left.\frac{\partial^{2} \bar{\phi}(x ; a)}{\partial a^{2}}\right|_{a=a_{0}} & =\frac{\ell}{1-\ell} \frac{W_{q}(x) W_{q}^{\prime \prime}\left(a_{0}\right) \bar{v}\left(a_{0}\right)}{\left[W_{q}\left(a_{0}\right)\right]^{2}}+\frac{\ell}{1-\ell} \frac{S q W_{q}(x) W_{q}^{\prime}\left(a_{0}\right)}{W_{q}\left(a_{0}\right)} \\
& =\frac{\ell}{1-\ell} \frac{Z_{q}(x)}{Z_{q}\left(a_{0}\right)}\left(\frac{1-\varphi Z_{q}\left(a_{0}\right)}{Z_{q}^{\prime}\left(a_{0}\right)} Z_{q}^{\prime \prime}\left(a_{0}\right)+\varphi Z_{q}^{\prime}\left(a_{0}\right)\right)=\frac{\ell}{1-\ell} \frac{Z_{q}(x)}{Z_{q}\left(a_{0}\right)} \bar{f}\left(a_{0}\right),
\end{aligned}
$$


where

$$
\bar{f}(a):=\frac{1-\varphi Z_{q}(a)}{Z_{q}^{\prime}(a)} Z_{q}^{\prime \prime}(a)+\varphi Z_{q}^{\prime}(a), \quad a \in(0, \infty)
$$

By (21), we have

$$
\bar{f}^{\prime}(a)=\left(1-\varphi Z_{q}(a)\right) \frac{W_{q}(a) W_{q}^{\prime \prime}(a)-\left(W_{q}^{\prime}(a)\right)^{2}}{\left(W_{q}(a)\right)^{2}}>0, \quad a \in(0, \infty) .
$$

Hence, by (44) and a similar argument as the one used in the proof of case $\mathbf{A}$ of Theorem 2.1, one can verify that the map $a \mapsto \bar{\phi}(x ; a)$ cannot have a local minimum point or saddle point in $[0, \infty)$. Indeed,

- if $a_{0}$ is a local minimum point of the map $a \mapsto \bar{\phi}(x ; a)$, then there should be $a_{0}^{\prime} \in\left(a_{0}, \infty\right)$ such that $\bar{f}\left(a_{0}\right) \geq 0$ and $\bar{f}\left(a_{0}^{\prime}\right) \leq 0$, which contradicts to (48);

- if $a_{0}$ is a saddle point of the map $a \mapsto \bar{\phi}(x ; a)$, then by $(21)$ and

$$
Z_{q}^{\prime \prime}\left(a_{0}\right)=-\frac{\varphi\left(Z_{q}^{\prime}\left(a_{0}\right)\right)^{2}}{1-\varphi Z_{q}\left(a_{0}\right)}, \quad \bar{V}^{\prime}\left(a_{0}\right)=\frac{1}{1-\varphi Z_{q}\left(a_{0}\right)},
$$

one can obtain $\bar{h}^{\prime}\left(a_{0}\right)=0$ and

$$
\begin{aligned}
\bar{h}^{\prime \prime}\left(a_{0}\right) & =\frac{Z_{q}^{\prime}\left(a_{0}\right) Z_{q}^{\prime \prime \prime}\left(a_{0}\right)\left(1-\varphi Z_{q}\left(a_{0}\right)\right)^{2}-\varphi^{2}\left[Z_{q}^{\prime}\left(a_{0}\right)\right]^{4}}{\left(1-\varphi Z_{q}\left(a_{0}\right)\right)\left[Z_{q}^{\prime}\left(a_{0}\right)\right]^{3} / Z_{q}\left(a_{0}\right)} \\
& >\frac{\left(Z_{q}^{\prime \prime}\left(a_{0}\right)\right)^{2}\left(1-\varphi Z_{q}\left(a_{0}\right)\right)^{2}-\varphi^{2}\left[Z_{q}^{\prime}\left(a_{0}\right)\right]^{4}}{\left(1-\varphi Z_{q}\left(a_{0}\right)\right)\left[Z_{q}^{\prime}\left(a_{0}\right)\right]^{3} / Z_{q}\left(a_{0}\right)}=0,
\end{aligned}
$$

implying that $\bar{h}(a)$ reaches a local minimum value of 0 at $a_{0}$. The above inequality holds because that $Z_{q}^{\prime}\left(a_{0}\right) Z_{q}^{\prime \prime \prime}\left(a_{0}\right)>\left(Z_{q}^{\prime \prime}\left(a_{0}\right)\right)^{2}$. In this case there must exist $a_{0}^{\prime} \in\left(a_{0}, \infty\right)$ such that $a_{0}$ is a maximum point or another saddle point of the map $a \mapsto \bar{\phi}(x ; a)$, i.e., $\bar{f}\left(a_{0}\right)=0$ and $\bar{f}\left(a_{0}^{\prime}\right) \leq 0$, which again contradicts to $(48)$.

We thus conclude that the equation (16) has at most one positive solution.

Finally, we shall examine the existence of any positive solution of the equation (16).

- When $\bar{v}(0)>\bar{V}(0)\left(1-\varphi Z_{q}(0)\right)$, by (44) one concludes that a positive solution $a^{+} \in(0, \infty)$ of (16) exists such that

$$
\bar{v}(a)>\bar{V}(a)\left(1-\varphi Z_{q}(a)\right), a \in\left[0, a^{+}\right) \quad \text { and } \quad \bar{v}(a)<\bar{V}(a)\left(1-\varphi Z_{q}(a)\right), a \in\left(a^{+}, \infty\right),
$$

i.e., the map $a \mapsto \bar{\phi}(x ; a)$ reaches its global maximum value at $a^{*}=a^{+}$. 
- When $\bar{v}(0)<\bar{V}(0)\left(1-\varphi Z_{q}(0)\right)$, then (16) has no positive solution and

$$
\bar{v}(a)<\bar{V}(a)\left(1-\varphi Z_{q}(a)\right), \quad a \in[0, \infty)
$$

i.e., the map $a \mapsto \bar{\phi}(x ; a)$ reaches its global maximum value at $a^{*}=0$.

- When $\bar{v}(0)=\bar{V}(0)\left(1-\varphi Z_{q}(0)\right)$, (49) holds true for all $a \in(0, \infty)$ and the map $a \mapsto \bar{\phi}(x ; a)$ reaches its global maximum value at $a^{*}=0$.

The proof is completed. 


\section{University Library}

\section{- M M I N E R VA A gateway to Melbourne's research publications}

Minerva Access is the Institutional Repository of The University of Melbourne

Author/s:

Wang, W;Wu, X;Chi, C

Title:

Optimal implementation delay of taxation with trade-off for spectrally negative Levy risk processes

Date:

2020-09-02

\section{Citation:}

Wang, W., Wu, X. \& Chi, C. (2020). Optimal implementation delay of taxation with trade-off for spectrally negative Levy risk processes. EUROPEAN ACTUARIAL JOURNAL, 11 (1), pp.285-317. https://doi.org/10.1007/s13385-020-00246-x.

Persistent Link:

http://hdl.handle.net/11343/253885 